\title{
Stéphanie Kaiser Das Abkommen über verarbeitete Landwirtschaftsprodukte: Bedeutung für die Schweiz
}


Das Europainstitut der Universität Basel ist ein rechts-, politik- und wirtschaftswissenschaftliches Zentrum für interdisziplinäre Lehre und Forschung zu europäischen Fragen. Das Institut bietet ein einjähriges, praxisbezogenes und interdisziplinäres Nachdiplomstudium zum Master of Advanced Studies in European Integration an, die Vertiefungsstudien Major in Conflict and Development und Major in International Business sowie neu auch ein massgeschneidertes Zertifikatsprogramm (Certificate of Advanced Studies). Daneben führt das Institut zusammen mit der Juristischen, der Wirtschaftswissenschaftlichen und der Philosophisch-Historischen Fakultät der Universität Basel einen Masterstudiengang European Studies durch. Es finden ausserdem regelmässig spezielle Weiterbildungskurse, Seminare und Vorträge statt. In der Forschung werden in Zusammenarbeit mit benachbarten Instituten sowohl fachspezifische wie multidisziplinäre Themen bearbeitet. Das Europainstitut ist als Ansprechpartner für Politik, Wirtschaft und Verwaltung beratend tätig. www.europa.unibas.ch

ISBN-13: 978-3-905751-27-7

ISBN-10: 3-905751-27-5

Stéphanie Kaiser erlangte 2006 ihren Master in internationalen Beziehungen am Genfer Hochschulinstitut für internationale Studien. Im September 2012 schloss sie berufsbegleitend ihren Master of Advanced Studies in europäischer Integration am Europainstitut der Universität Basel ab. Gegenwärtig arbeitet sie als stv. Ressortleiterin Exportkontrollpolitik in der Direktion für Aussenwirtschaft im Staatssekretariat für Wirtschaft SECO. Die vorliegende Arbeit ist eine leicht überarbeitete Version ihrer Diplomarbeit zur Erlangung des Titels "Master of Advanced Studies in European Integration, Major in International Business" und wurde von Prof. Dr. Rolf Weder und Prof. Dr. Christa Tobler betreut. 
BASLERSCHRIFTEN zur europäischen Integration Nr. 102

Stéphanie Kaiser Das Abkommen über verarbeitete Landwirtschaftsprodukte: Bedeutung für die Schweiz 


\section{Inhaltsverzeichnis}

1. Einleitung

2. Protokoll Nr. 2 - worum geht es?

3. Aktuelle Herausforderungen in Bezug auf das Protokoll Nr. 2

3.1 Multilaterale Entwicklungen im Rahmen der WTO

3.2 Budgetknappheit des Schoggigesetzes

3.3 Stand der Beziehungen Schweiz-EU in Bezug auf das

Protokoll Nr. 2

3.4 Fazit

4. Wirkungen von Importzöllen und Exportsubventionen

4.1 Wirkungen von Importzöllen

4.2 Wirkungen von Exportsubventionen

5. Bedeutung des Protokolls Nr. 2 für die Schweiz

5.1 Bedeutung des Handels im Rahmen des Protokolls Nr. 2

5.2 Bedeutung für die Landwirtschaft 27

5.3 Bedeutung für die Nahrungsmittelindustrie 31

5.4 Gesamtvolkswirtschaftliche Bedeutung für die Schweiz 34

5.5 Fazit

6. Evaluation Handlungsdruck und Skizzierung möglicher Szenarien

7. Schlussfolgerungen

Literaturverzeichnis 


\section{Abbildungsverzeichnis}

Abb. 1: Wirkungen von Importzöllen 20

Abb. 2: Wirkungen von Exportsubventionen 21

Abb. 3: Handel im Rahmen des Protokolls Nr. 2

Abb. 4: Handel der Freihandelsprodukte 26

Abb. 5: Handel der preisausgleichberechtigten Produkte 26

\section{Tabellenverzeichnis}

Tab. 1: Schoggigesetz-Budget 


\section{Einleitung}

Der Handel mit landwirtschaftlichen Verarbeitungsprodukten zwischen der Schweiz und der EU wird im Rahmen des Protokolls Nr. 2 zum Freihandelsabkommen (FHA) von 1972 geregelt. ${ }^{1}$ Die Handelsbilanz der Schweiz mit der EU im Rahmen des Protokolls Nr. 2 ist wertmässig positiv. Die Schweizer Exporte beliefen sich im Jahr 2011 auf rund 3,7 Mrd. Franken und übertrafen damit die Importe um rund 1 Mrd. Franken. ${ }^{2}$ Gleichzeitig steht das Protokoll Nr. 2 von verschiedenen Seiten unter Druck, was seine Zukunft in Frage stellt. Das Grundproblem stellen die Ausfuhrbeiträge im Rahmen des Preisausgleichmechanismus dar.

Das Protokoll Nr. 2 wurde im Abkommen über landwirtschaftliche Verarbeitungsprodukte im Rahmen der Bilateralen Verhandlungen II (2004) revidiert. ${ }^{3}$ Die vorliegende Arbeit konzentriert sich auf das revidierte Protokoll Nr. 2 und analysiert dessen Bedeutung für die Schweiz. Hierfür wird differenziert zwischen den preisausgleichberechtigen Produkten und den Freihandelsprodukten, welche im Geltungsbereich des Protokolls liegen. Die Arbeit zeigt auf, dass die gegenseitige Zollfreiheit für die Freihandelsprodukte aus volkswirtschaftlichter Perspektive positiv ist. Die Preisausgleichmassnahmen für die preisausgleichberechtigten Produkte sind hingegen in erster Linie für die Landwirtschaft von Bedeutung. Gesamtvolkswirtschaftlich betrachtet wäre die Alternative eines umfassenden Freihandels

1 Protokoll Nr. 2 vom 22. Juli 1972 über bestimmte landwirtschaftliche Verarbeitungserzeugnisse (SR 0.632.401.2).

2 Statistische Angaben von Peter Huber (SECO), 2012. (Vgl. Kapitel 4.1)

3 Abkommen vom 26. Oktober 2004 zwischen der Schweizerischen Eidgenossenschaft und der Europäischen Gemeinschaft zur Änderung des Abkommens zwischen der Schweizerischen Eidgenossenschaft und der Europäischen Wirtschaftsgemeinschaft vom 22. Juli 1972 in Bezug auf die Bestimmungen über landwirtschaftliche Verarbeitungserzeugnisse (SR 0.632.401.23). 
im Agrar- und Lebensmittelbereich mit der EU wohlfahrtssteigernd, womit auch die Preisausgleichmassnahmen überflüssig würden. Aufgrund verschiedener Faktoren ist ein solcher Freihandel mit der EU, oder eine vergleichbare Liberalisierung auf multilateraler Ebene, jedoch nicht absehbar. Gleichzeitig besteht auf internationaler Ebene der Druck, alle Exportsubventionen, worunter auch die Ausfuhrbeiträge im Rahmen des Preisausgleichmechanismus fallen, abzubauen. Somit dürften in Zukunft Alternativen in Form einer Ausweitung des aktiven Veredelungsverkehrs sowie möglicherweise der brancheninternen privatrechtlichen Massnahmen weiter an Bedeutung gewinnen.

Diese Arbeit ist wie folgt strukturiert: In einem ersten Schritt werden die wichtigsten Aspekte des Protokolls Nr. 2 kurz präsentiert. Danach werden verschiedene Herausforderungen in Bezug auf das Protokoll Nr. 2 diskutiert und die Wirkungen von Importzöllen und Exportsubventionen aus einer volkswirtschaftlichen Perspektive beleuchtet. In Kapitel 5 wird basierend darauf die Bedeutung des Protokolls Nr. 2 für die Schweiz analysiert. Hierfür wird zunächst die Entwicklung des Handels im Rahmen des Protokolls Nr. 2 dargestellt. Danach wird die Bedeutung dieses Protokolls für die beiden hauptsächlich betroffenen Sektoren, die Landwirtschaft und die Nahrungsmittelindustrie, untersucht. Schliesslich werden die gesamtwirtschaftliche Bedeutung für die Schweiz erörtert und der Handlungsdruck sowie mögliche Zukunftsszenarien dargestellt. 


\section{Protokoll Nr. 2 - worum geht es?}

Das Protokoll Nr. 2 zum Freihandelsabkommen (FHA) zwischen der Schweiz und der EU von 1972 regelt den Handel mit landwirtschaftlichen Verarbeitungsprodukten. ${ }^{4}$ Das FHA legt die Grundlage für den Freihandel mit industriellen Gütern, während die Landwirtschaftsprodukte vom Geltungsbereich des Abkommens ausgeschlossen sind. Die landwirtschaftlichen Verarbeitungsprodukte wiederum, wie z.B. Schokolade und Teigwaren, nehmen eine Sonderstellung zwischen Industrie (Freihandel) und Landwirtschaft (Agrarschutz) ein, da sie sowohl einen Agrarrohstoffteil als auch einen industriellen Verarbeitungsteil umfassen. Basierend auf dem Protokoll Nr. 2 wurden die Zölle auf dem industriellen Verarbeitungsteil dieser Produkte aufgehoben und für den Agrarrohstoffteil ein so genannter Preisausgleichmechanismus eingeführt. ${ }^{5}$

Die Logik des Preisausgleichmechanismus liegt darin, den Wettbewerbsnachteil hoher Agrarrohstoffpreise durch entsprechende Einfuhrabgaben (Importzölle) und Ausfuhrbeiträge (Exportsubventionen) auszugleichen. Einfuhrabgaben verteuern die in den importierten Produkten enthaltenen Agrarrohstoffe, während die Ausfuhrbeiträge die in den exportierten Produkten enthaltenen Agrarrohstoffe verbilligen. ${ }^{6}$ Im Schweizerischen Landesrecht stützen sich diese Preis-

4 Protokoll Nr. 2 vom 22. Juli 1972 über bestimmte landwirtschaftliche Verarbeitungserzeugnisse (SR 0.632.401.2).

5 Roth, Thomas (2004): „Abkommen über verarbeitete Landwirtschaftsprodukte eine neue Etappe in den Freihandelsbeziehungen mit der EU“. Die Volkswirtschaft 9.2004. Sowie Integrationsbüro EDA/EVD (2011): „Landwirtschaftliche Verarbeitungsprodukte: Informationsblatt" 8.2011.

6 Roth, Thomas (2004): „Abkommen über verarbeitete Landwirtschaftsprodukte eine neue Etappe in den Freihandelsbeziehungen mit der EU“. Die Volkswirtschaft 9.2004. Sowie Integrationsbüro EDA/EVD (2011): „Landwirtschaftliche Verarbeitungsprodukte: Informationsblatt“ 8.2011 . 
ausgleichmassnahmen auf das so genannte "Schoggigesetz". ${ }^{7}$ Das Schoggigesetz kommt sowohl im Rahmen des Protokolls Nr. 2, als auch im Rahmen des Handels zwischen der Schweiz und weiterer Handelspartner zur Anwendung.

Die Schweiz verfolgt mit dem Preisausgleichmechanismus im Rahmen des Schoggigesetzes das Ziel, eine wettbewerbsfähige Schweizer Nahrungsmittelindustrie zu ermöglichen, bei gleichzeitig hohem Grenzschutz in der Landwirtschaft. ${ }^{8}$ Es soll sichergestellt werden, dass die Schweizer Nahrungsmittelindustrie inländische Agrarrohstoffe verarbeitet und dabei gleichzeitig auf dem inländischen Markt (durch Einfuhrabgaben) wie ausländischen Markt (durch Ausfuhrbeiträge) wettbewerbsfähig ist. Preisausgleichmassnahmen kommen nicht nur für Agrarrohstoffe (z.B. Milch, Getreide) zur Anwendung, sondern auch für Zwischenprodukte der 1. Verarbeitungsstufe (z.B. Butter, Mehl). ${ }^{9}$ Von Bedeutung ist der Preisausgleich insbesondere für verarbeitete Milch- und Getreideprodukte. ${ }^{10}$ Auch wenn das Schoggigesetz nur indirekt ein agrarpolitisches Instrument ist, so hat es zum Ziel, agrarpolitisch bedingte Preisunterschiede auszugleichen. ${ }^{11}$ Das Schoggigesetz kann somit als eine Konsequenz der Schweizer Agrarpolitik betrachtet werden.

Im Rahmen der Bilateralen Verhandlungen II (2004) wurde das Pro-

7 Bundesgesetz vom 13. Dezember 1974 über die Ein- und Ausfuhr von Erzeugnissen aus Landwirtschaftsprodukten (SR 632.111.72).

8 Tinner, Willy (2006) „Landwirtschaftliche Verarbeitungserzeugnisse: Würdigung des Abkommens“, in Kaddous, Christine / Jametti Greiner, Monique (Hrsg.): Bilaterale Abkommen II Schweiz-EU und andere neue Abkommen. Genf, Basel (etc.): Helbling \& Lichtenhahn (etc.), S. 657-674.

9 Verordnung über die Ausfuhrbeiträge für Erzeugnisse aus Landwirtschaftsprodukten vom 23. November 2011 (SR 632.111.723), Art.1.

10 Persönliche Auskunft von Markus Schlagenhof (SECO), 31.5.2012.

11 BLW:,,Agrarbericht 2011“, Bundesamt für Landwirtschaft, Bern. S. 107. 
tokoll Nr. 2 im Abkommen über landwirtschaftliche Verarbeitungsprodukte revidiert. ${ }^{12}$ Mit dem Abkommen konnten die Verhandlungsziele der Schweiz vollumfänglich erreicht werden. ${ }^{13}$ Für die vorliegende Arbeit sind insbesondere die dadurch erzielte Vereinfachung des Preisausgleichmechanismus und die Erweiterung des Produktdeckungsbereichs von Bedeutung:

Die Vereinfachung des Preisausgleichmechanismus hatte die so genannte Nettopreis-Kompensation zur Folge. Vor der Revision waren die Preisausgleichmassnahmen der Schweiz und der EU auf das Weltmarktpreisniveau ausgerichtet. Mit der Nettopreis-Kompensation wird nun nicht mehr die Differenz zum Weltmarktpreis als Referenz für die Berechnung der Preisausgleichmassnahmen herbeigezogen, sondern nur noch die kleinere Differenz zwischen den Schweizer und den EU-Agrarrohstoffpreisen. Da die Agrarrohstoffpreise in der Schweiz in der Regel höher sind als in der EU, trifft nur noch die Schweiz Preisausgleichmassnahmen. Gleichzeitig werden dadurch die schweizerischen Ausfuhrbeiträge und Einfuhrabgaben reduziert. ${ }^{14}$

Im Rahmen der Erweiterung des Produktdeckungsbereichs wurde die Liste der Produkte erweitert, für welche gegenseitige Zollfreiheit gilt. Für die Schweiz von Interesse war insbesondere der Freihandel für Nahrungsergänzungsmittel, Phytopharmaka, Röstkaffee und löslichen Kaffee. Die Aufnahme von u.a. Spirituosen, Hefe und Es-

12 Abkommen vom 26. Oktober 2004 zwischen der Schweizerischen Eidgenossenschaft und der Europäischen Gemeinschaft zur Änderung des Abkommens zwischen der Schweizerischen Eidgenossenschaft und der Europäischen Wirtschaftsgemeinschaft vom 22. Juli 1972 in Bezug auf die Bestimmungen über landwirtschaftliche Verarbeitungserzeugnisse (SR 0.632.401.23).

13 Botschaft zur Genehmigung der bilateralen Abkommen zwischen der Schweiz und der Europäischen Union, einschliesslich der Erlasse zur Umsetzung der Abkommen („Bilaterale II“) vom 1. Oktober 2004. S. 6035.

14 Ibid. S. 5996-5997. 
sig entsprach den Interessen der EU. Diese Freihandelsprodukte unterliegen nicht dem Preisausgleich, da sie keine agrarpolitisch relevanten Agrarrohstoffe (wie u.a. Milch, Getreide) beinhalten. ${ }^{15}$

Das Protokoll Nr. 2 umfasst zwei Produktekategorien. Für die landwirtschaftlichen Verarbeitungsprodukte der Tabelle I kommt der Preisausgleichmechanismus zur Anwendung (preisausgleichberechtigte Produkte), während für die landwirtschaftlichen Verarbeitungsprodukte der Tabelle II gegenseitige Zollfreiheit gilt (Freihandelsprodukte). ${ }^{16}$

\section{Aktuelle Herausforderungen in Bezug auf das Protokoll Nr. 2}

Das Protokoll Nr. 2 steht heute von verschiedenen Seiten unter Druck, was seine Zukunft in Frage stellt. Das Grundproblem stellen die Ausfuhrbeiträge im Rahmen des Preisausgleichmechanismus dar.

Der Ursprung des Problems liegt darin, dass sich Exportsubventionen zunehmend zu einem international inakzeptablen handelspolitischen Instrument entwickelt haben. Die Ausfuhrbeiträge im Rahmen des Preisausgleichmechanismus entsprechen einer Exportsubvention, auch wenn das Ziel lediglich der Ausgleich der Preise der Agrarrohstoffe ist, um den durch den hohen Grenzschutz verursachten Wettbewerbsnachteil aufzuheben und nicht die Schaffung eines darüber hinausgehenden Wettbewerbsvorteils für die Schweizer Nahrungsmittelindustrie. ${ }^{17}$

15 Roth, Thomas (2004): „Abkommen über verarbeitete Landwirtschaftsprodukte eine neue Etappe in den Freihandelsbeziehungen mit der EU“. Die Volkswirtschaft 9.2004.

16 Protokoll Nr. 2 vom 22. Juli 1972 über bestimmte landwirtschaftliche Verarbeitungserzeugnisse (SR 0.632.401.2), Tabelle I und II.

17 Tinner, Willy (2006) „Landwirtschaftliche Verarbeitungserzeugnisse: Würdigung 
Im Folgenden wird auf verschiedene aktuelle Herausforderungen betreffend die Zukunft des Protokolls Nr. 2 eingegangen. Beleuchtet werden die Entwicklungen auf multilateraler Ebene im Rahmen der WTO, die Budgetknappheit des Schoggigesetzes sowie der aktuelle Beziehungsstand zwischen der Schweiz und der EU hinsichtlich des Protokolls Nr. 2.

\subsection{Multilaterale Entwicklungen im Rahmen der WTO}

Am Ministertreffen in Hongkong im Jahr 2005 beschlossen die WTOMitglieder alle Exportsubventionen bis Ende 2013 abzuschaffen und die Höhe dieser Beiträge vor diesem Zeitpunkt schrittweise und substanziell zu kürzen. Die Implementierung dieses Beschlusses setzt allerdings den Abschluss der Doha-Runde voraus. ${ }^{18}$ Bereits im Rahmen der Uruguay-Runde von 1994 verpflichteten sich die WTOMitglieder darauf, die Ausfuhrbeiträge für landwirtschaftliche Verarbeitungsprodukte zu beschränken. Basierend darauf hält die WTOVerpflichtungsliste der Schweiz fest, dass sie jährlich maximal 114,9 Mio. Franken für Exportsubventionen auf landwirtschaftliche Verarbeitungsprodukte entrichten darf. ${ }^{19}$

Die Zukunft der WTO Doha-Runde und damit jene der Agrarverhandlungen ist ungewiss. Die Themen liegen jedoch weiterhin auf dem Tisch. Die Exportsubventionen stehen für ihre verzerrende Wirkung

des Abkommens", in Kaddous, Christine / Jametti Greiner, Monique (Hrsg.): Bilaterale Abkommen II Schweiz-EU und andere neue Abkommen. Genf, Basel (etc.): Helbling \& Lichtenhahn (etc.), S. 671.

18 WTO: "Ministerial Declaration adopted on 18 December 2005“, Paragraph 6, sowie Interpellation 10.3735 Grin Jean-Pierre: „Zukunft des „Schoggigesetzes“, Antwort des Bundesrates vom 17.11.2010.

19 WTO: "Goods schedule annexed to Marrakesh Protocol, Liste LIX (59)", CHE4201. 
auf die internationalen Agrarmärkte unter breiter Kritik und die überwiegende Mehrzahl der WTO-Mitgliedstaaten richtet keine solchen Subventionen mehr aus. Die Schweiz selbst hat auf Ende 2009 ihre Exportsubventionen für Agrarrohstoffe abgeschafft. Vor diesem Hintergrund bleibt der Druck, die Exportsubventionen gänzlich abzuschaffen, bestehen. ${ }^{20}$

\subsection{Budgetknappheit des Schoggigesetzes}

Abgesehen vom internationalen Druck auf die Exportsubventionen stellt die Budgetknappheit des Schoggigesetzes und die dadurch ungenügend zur Verfügung stehenden Ausfuhrbeiträge ein weiteres Hindernis für die Anwendung des Schoggigesetzes und somit des Protokolls Nr. 2 dar. Im Jahr 2011 wurden 65\% der Ausfuhrbeiträge des Schoggigesetzes im Rahmen des Protokolls Nr. 2 für Exporte in die EU eingesetzt. ${ }^{21}$

Das ordentliche Budget des Schoggigesetzes hat sich seit 2004 sukzessive verkleinert, wobei aber seit 2009 verschiedene Nachkredite (NK) sowie ein Zusatz im Rahmen der Bekämpfung der Frankenstärke gewährt wurden (vgl. Tabelle 1). In den Jahren 2007 und 2008 war der Mittelbedarf aufgrund hoher Weltmarktpreise für Rohstoffe insgesamt kleiner. Seit dem 1. Mai 2010 ist es jedoch erstmals teilweise nicht mehr möglich, die gesamte Preisdifferenz durch Ausfuhrbeiträge auszugleichen, da der Mittelbedarf das Budget übersteigt. Bis anhin waren Teilkompensationen der Preisausgleichdifferenz von teilweise nur noch $50 \%$ die Folge. Für das laufende Jahr 2012 sind wiederum 70 Mio. Franken gesprochen worden. ${ }^{22}$

20 Persönliche Auskunft von Remigi Winzap (SECO), 30.3.2012.

21 Persönliche Auskunft von Peter Huber (SECO), 6.6.2012.

22 Ibid. 


\begin{tabular}{|c|c|c|c|c|c|c|c|}
\hline 2004 & 2005 & 2006 & 2007 & 2008 & 2009 & 2010 & 2011 \\
\hline 114,9 & 90 & 90 & 79,2 & 75 & 93 (inkl. 18 & 77,6 (inkl. 7,6 & 76,3 (inkl. 6,3 \\
& & & & & NK) & NK) & Frankenstärke) \\
\hline
\end{tabular}

Tab. 1: Schoggigesetz-Budget in Mio. Franken. Quelle: Peter Huber (SECO), 2012.

Die sukzessive Verkleinerung des ordentlichen Budgets lässt sich zumindest teilweise durch die in 3.1 beschriebene Verpflichtung der Schweiz im Rahmen der WTO-Ministererklärung von Hongkong von 2005 erklären. ${ }^{23}$ Dank der im Abkommen über landwirtschaftliche Verarbeitungsprodukte ausgehandelten Netto-Preiskompensation konnte der Mittelbedarf des Schoggigesetzes verkleinert werden. Heute ist er jedoch aufgrund der Zunahme bei den Exporten wieder angestiegen. ${ }^{24}$

Als Folge der nicht ausreichenden Budgetmittel kommen gestützt auf das Zollgesetz privatrechtliche Massnahmen sowie der aktive Veredelungsverkehr zur Anwendung. ${ }^{25}$ Bei den privatrechtlichen Massnahmen geht es darum, dass sich die Landwirtschaft und teilweise die 1. Verarbeitungsstufe (z.B. Milchpulverhersteller oder Müller) organisiert um die verbleibende Preisdifferenz von inländischen Agrarrohstoffen (oder Zwischenprodukten), welche für Exportprodukte bestimmt sind, zu kompensieren. ${ }^{26}$ Die Folge ist eine Verbilligung der inländischen Agrarrohstoffe, welche für Exportprodukte bestimmt sind. Wenn aber im Inland die benötigten Agrarrohstoffe nicht genügend und ohne Preisnachteil verfügbar sind und diese privatrechtlichen Massnahmen nicht zur Anwendung kommen, besteht gestützt auf

23 Interpellation 10.3735 Grin Jean-Pierre: „Zukunft des „Schoggigesetzes“, Antwort des Bundesrates vom 17.11.2010.

24 Persönliche Auskunft von Markus Schlagenhof (SECO), 31.5.2012.

25 Zollgesetz vom 18. März 2005 (SR 631.0), Art.12, Abs. 3.

26 Persönliche Auskunft von Jacques Chavaz und Kilian Greter (beide BLW), 10.7.2012. 
das Zollgesetz Anrecht auf den aktiven Veredelungsverkehr. ${ }^{27}$ Im Rahmen des aktiven Veredelungsverkehrs kann die verarbeitende Nahrungsmittelindustrie die für ihre Exportprodukte benötigten Agrarrohstoffe zollfrei in die Schweiz importieren. ${ }^{28}$

Obwohl seit Mai 2010 die Ausfuhrbeiträge des Bundes nicht mehr immer ausreichen, konnte kein markanter Anstieg des aktiven Veredelungsverkehrs beobachtet werden. ${ }^{29}$ Dies lässt sich dadurch erklären, dass die beiden hauptsächlich durch die Budgetknappheit betroffenen Sektoren, die Milchbranche und die Getreidebranche, privatrechtliche Massnahmen ergreifen. ${ }^{30}$ Damit soll verhindert werden, durch den aktiven Veredelungsverkehr Marktanteile an ausländische Agrarrohstoffanbieter zu verlieren. So hat beispielsweise die Branchenorganisation Milch (BO Milch) im Jahr 2010 einen Interventionsfond beschlossen. Der Fonds wird durch einen Beitrag sämtlicher Milchproduzenten und Molkereimilchverarbeiter von 0,5 Rp./kg finanziert und kompensiert die Erstattungslücke bei den öffentlichen Ausfuhrbeiträgen teilweise. ${ }^{31}$

Diese Situation der ungenügend zur Verfügung stehenden Ausfuhrbeiträge führt zu Differenzen innerhalb der Branche über den Beteiligungsgrad an diesen privatrechtlichen Massnahmen. ${ }^{32}$ Zur Branche zählen die Landwirtschaft (Primärproduzent), die Verarbei-

27 Zollgesetz vom 18. März 2005 (SR 631.0), Art.12, Abs. 3.

28 Siehe für weitergehende Informationen Eidgenössische Zollverwaltung EZV, http://www.ezv.admin.ch.

29 Persönliche Auskunft von Jacques Chavaz und Kilian Greter (beide BLW), 10.7.2012.

$30 \mathrm{lbid}$.

31 BO Milch: „Interventionsfond“, http://www.ip-lait.ch., zuletzt besucht am 21.7.2012. 32 Persönliche Auskunft von Mathias Bergundthal (Nestlé Schweiz), 5.7.2012. Siehe auch Wyss-Aerni, Roland (2008): „Rohstoffverbilligung kommt die Bauern teuer", Landwirtschaftlicher Informationsdienst LID, 31.3.2008. 
ter der 1. Stufe (z.B. Milchpulverhersteller) und die Verarbeiter der 2. Stufe (die verarbeitende Nahrungsmittelindustrie). ${ }^{33}$ Insbesondere die Landwirtschaft selbst sieht sich bestraft, da sie einen Teil des Ausgleichs der Rohstoffpreisdifferenz selbst bezahlen muss. ${ }^{34}$ Auf der anderen Seite bemängelt die verarbeitende Nahrungsmittelindustrie im Zusammenhang mit der knappen Budgetsituation in erster Linie eine gewisse Planungs- und Rechtsunsicherheit. ${ }^{35}$

\subsection{Stand der Beziehungen Schweiz-EU in Bezug auf das Proto- koll Nr. 2}

Neben den Entwicklungen auf multilateraler Ebene betreffend den Abbau von Exportsubventionen und der Budgetknappheit des Schoggigesetzes, bildet die Beziehung Schweiz-EU in Bezug auf die Umsetzung des Protokolls Nr. 2 die dritte Front, welche es zu beachten gilt.

Die EU ist der Ansicht, dass aufgrund von Verpflichtungen im WTOAgrarabkommen die Schweizer Ausfuhrbeiträge für landwirtschaftliche Verarbeitungsprodukte bereits heute nicht WTO-konform seien. ${ }^{36}$ Des Weiteren verzichtet die EU selbst im Rahmen ihrer Freihandelsabkommen seit geraumer Zeit auf Preisausgleichmassnahmen für landwirtschaftliche Verarbeitungsprodukte und gewährt ihren

33 Persönliche Auskunft von Mathias Bergundthal (Nestlé Schweiz), 5.7.2012.

34 Wyss-Aerni, Roland (2008): "Rohstoffverbilligung kommt die Bauern teuer", Landwirtschaftlicher Informationsdienst LID, 31.3.2008.

35 Persönliche Auskunft von Mathias Bergundthal (Nestlé Schweiz), 5.7.2012, sowie von Franz Urs Schmid (Fial), 11.7.2012.

36 Schweizer Bauer (2009): „Einigung bei Rohstoffpreisausgleich“, 19.12.2009, sowie Neue Zürcher Zeitung NZZ (2009): „Ehrgeizige Verhandlungsziele für EUAgrarabkommen", 27.6.2009. 
Handelspartnern weitgehend Zollfreiheit. ${ }^{37}$ Die Schweiz teilt die Interpretation der EU betreffend die WTO-Widrigkeit ihrer Ausfuhrbeiträge allerdings nicht. ${ }^{38}$

Durch die 2008 eröffneten Verhandlungen über ein Freihandelsabkommen im Agrar- und Lebensmittelbereich (FHAL) konnten die Meinungsverschiedenheiten zwischen der Schweiz und der EU hinsichtlich des Protokolls Nr. 2 in der Vergangenheit zur Seite geschoben werden. ${ }^{39}$ Das FHAL sieht eine umfassende Liberalisierung des Handels mit Agrargütern und Lebensmitteln vor, womit auch das Protokoll Nr. 2 abgeschafft würde. ${ }^{40}$

Diese Verhandlungen sind aber zwischenzeitlich aufgrund verschiedener Faktoren ins Stocken geraten. Nebst Differenzen über den Verhandlungsgegenstand werden die Verhandlungen von den allgemeinen institutionellen Fragen überschattet. ${ }^{41}$ Ferner sind die Verhandlungen in der Schweiz innenpolitisch unter Druck geraten. Im März 2012 wurde eine Motion vom Parlament verabschiedet, welche verlangt, die Verhandlungen mit der EU im Agrar- und Lebensmittelbereich zu stoppen, solange kein Abschluss der WTO Doha-Runde zustande kommt. ${ }^{42}$ Gleichzeitig wurde eine Motion vom Ständerat angenommen, welche den Bundesrat beauftragt, eine Standortbestimmung der Verhandlungen vorzunehmen und Alternativen aufzuzeigen. ${ }^{43}$

37 Persönliche Auskunft von Peter Huber (SECO), 6.6.2012.

38 Schweizer Bauer (2009): „Einigung bei Rohstoffpreisausgleich“, 19.12.2009, sowie Neue Zürcher Zeitung NZZ (2009): „Ehrgeizige Verhandlungsziele für EUAgrarabkommen“, 27.6.2009.

39 lbid.

40 BLW:,Agrarbericht 2011“, Bundesamt für Landwirtschaft, Bern. S. 191.

41 lbid. S. 186.

42 Motion 10.3818 Darbellay Christophe: „Verhandlungen mit der EU über ein Freihandelsabkommen im Agrar- und Lebensmittelbereich stoppen“, eingereicht am 1.10.2010.

43 Motion 12.3014 Kommission für Wirtschaft und Abgaben SR: „Kontrollierte Öffnung im Agrarbereich statt Verhandlungsverbot", eingereicht am 14.2.2012. 


\subsection{Fazit}

Es überrascht kaum, dass heute das Protokoll Nr. 2 unter Druck steht und dies hauptsächlich aufgrund der darin vorgesehenen Exportsubventionen. Bereits in der Würdigung des Abkommens über landwirtschaftliche Verarbeitungsprodukte hielt der damalige Leiter der Schweizerischen Verhandlungsdelegation fest, dass die dauerhafte Lösung darin bestehen würde, in das Freihandelsabkommen zwischen der Schweiz und der EU (damals EG) auch den gesamten Agrarbereich einzuschliessen. ${ }^{44}$

Sollte den Verhandlungen über einen Freihandel im Agrar- und Lebensmittelbereich nicht bald neues Leben eingehaucht werden, muss damit gerechnet werden, dass der Druck auf das Protokoll Nr. 2 seitens der EU steigen wird. ${ }^{45}$

\section{Wirkungen von Importzöllen und Exportsubventionen}

In diesem Kapitel werden die Wirkungen von Importzöllen und Exportsubventionen aus volkswirtschaftlicher Perspektive beleuchtet. Der Fokus liegt auf den Konsequenzen für das Land selbst, welches sich dieser handelspolitischen Instrumente bedient.

Dieses Verständnis ist für die Analyse der Bedeutung des Protokolls Nr. 2 für die Schweiz im darauffolgenden Kapitel hilfreich.

44 Tinner, Willy (2006) „Landwirtschaftliche Verarbeitungserzeugnisse: Würdigung des Abkommens", in Kaddous, Christine / Jametti Greiner, Monique (Hrsg.): Bilaterale Abkommen II Schweiz-EU und andere neue Abkommen. Genf, Basel (etc.): Helbling \& Lichtenhahn (etc.), S. 674.

45 Vgl. Aussage von Markus Schlagenhof (SECO) im Schweizer Bauer (2009): „Einigung bei Rohstoffpreisausgleich“, 19.12.2009. 


\subsection{Wirkungen von Importzöllen ${ }^{46}$}

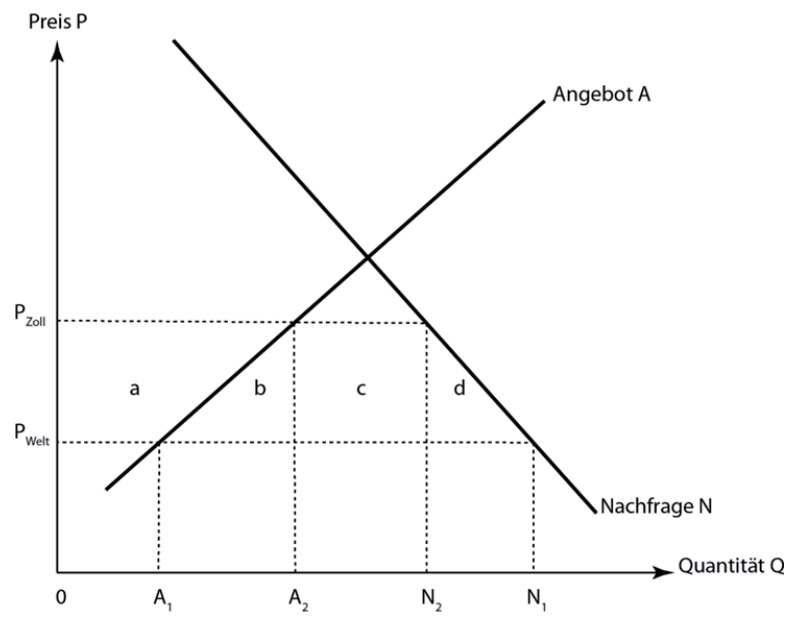

Abb. 1: Wirkungen von Importzöllen. Quelle: In Anlehnung an Krugman, Paul / Obstfeld, Maurice (2000).

Abbildung 1 stellt ein kleines Land mit inländischer Angebots- und Nachfragekurve dar. Ein kleines Land ist nicht in der Lage den Auslandpreis zu verändern und sieht sich dem gegebenen Weltmarktpreis $\mathrm{P}_{\text {welt }}$ gegenüber.

Bei Freihandel ist der Inlandspreis gleich dem Weltmarktpreis $P_{\text {well: }}$ Im Inland wird die Quantität $\mathrm{A}_{1}$ produziert und die Quantität $\mathrm{A}_{1} \mathrm{~N}_{1}$ wird importiert. Wenn dieses Land nun einen Importzoll einführt, steigt der inländische Preis um den Betrag des Zolls auf $P_{\text {zoll }}$. Die Wirkungen dieses Importzolles im Inland sind die folgenden: Der Konsum verringert sich von $\mathrm{N}_{1}$ auf $\mathrm{N}_{2}$ aufgrund der höheren Preise. Die Produktion vergrössert sich von $A_{1}$ auf $A_{2}$ ebenfalls aufgrund der höheren 46 Basiert auf Krugman, Paul / Obstfeld, Maurice (2000): „International Economics, Theory and Policy, Fifth Edition“, Addison-Wesley. S. 190-198. 
Preise. Es kommt dadurch zu einer Umverteilung der Wohlfahrt von Konsumenten zu Produzenten. Die Konsumentenrente sinkt um den Betrag von $a+b+c+d$. Die Produzentenrente dagegen steigt um den Betrag von a. Die Importe verringern sich von $A_{1} N_{1}$ auf $A_{2} N_{2}$. Die Staatseinnahmen der Zolleinnahmen belaufen sich auf c (Betrag des Zolls multipliziert mit der Importmenge $\mathrm{A}_{2} \mathrm{~N}_{2}$ ). Der resultierende Wohlfahrtsverlust umfasst den Betrag $b+d$. Dieser Effizienzverlust ist das Resultat der Verzerrung der Produktions- und Konsumanreize durch den Zoll. Die Einführung eines Importzolls durch ein kleines Land ist für das Land gesamtvolkswirtschaftlich gesehen wohlfahrtsmindernd.

Nachfolgend werden die gleichen Überlegungen für den Fall einer Exportsubvention durchgespielt.

\subsection{Wirkungen von Exportsubventionen ${ }^{47}$}

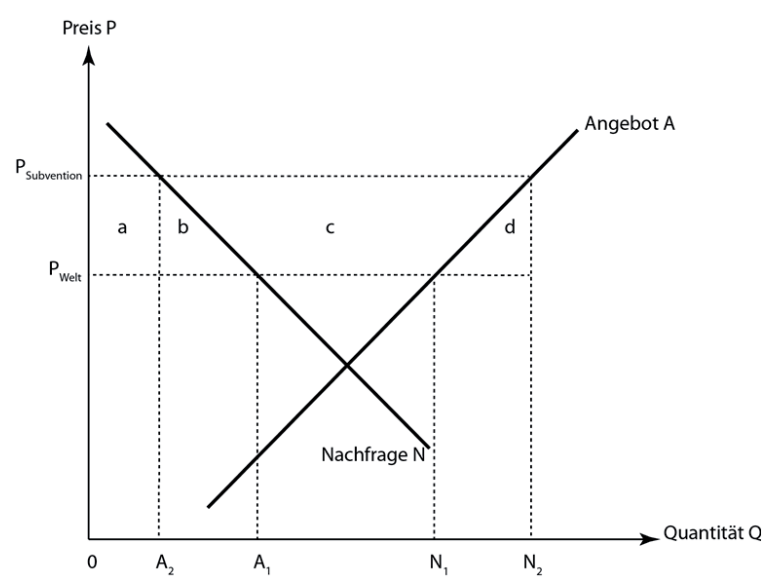

Abb. 2: Wirkungen von Exportsubventionen. Quelle: In Anlehnung an Krugman, Paul / Obstfeld, Maurice (2000).

47 Basiert auf Krugman, Paul / Obstfeld, Maurice (2000): „International Economics, Theory and Policy, Fifth Edition“, Addison-Wesley. S. 198-199. 
Auch die Abbildung 2 stellt ein kleines Land dar, welches den Weltmarktpreis $P_{\text {welt }}$ nicht beeinflussen kann. Angebot $A$ und Nachfrage $N$ stellen die inländische Angebots- und Nachfragekurve dar.

Bei Freihandel ist der Inlandspreis gleich dem Weltmarktpreis $P_{\text {Welt: }}$ Das Land exportiert die überschüssige Menge $A_{1} N_{1}$. Im Falle einer Exportsubvention steigt der inländische Preis um den Betrag der Exportsubvention auf $P_{\text {subvention. }}$. Die Wirkungen dieser Exportsubvention im Inland sind die folgenden: Der Export vergrössert sich auf $\mathrm{A}_{2} \mathrm{~N}_{2}$. Die Konsumentenrente sinkt um den Betrag von $a+b$, während die Produzentenrente um den Betrag von $a+b+c$ steigt. Erneut kommt es zu einer Umverteilung der Wohlfahrt von Konsumenten zu Produzenten. Die Kosten für den Staat ergeben sich aus der Exportmenge multipliziert mit der Exportsubvention, was dem Betrag von $b+c+d$ entspricht. Der aus der Exportsubvention resultierende Wohlfahrtsverlust beläuft sich auf den Betrag von $b+d$.

\subsection{Fazit}

Welche Folgerungen können aus der obenstehenden theoretischen Analyse von Importzöllen und Exportsubventionen auf den Preisausgleichmechanismus im Rahmen des Protokolls Nr. 2 und der ihm zu Grunde liegenden Grenzschutz geschlossen werden?

Die Analyse der Wirkungen von Importzöllen für ein kleines Land kann auf den hohen Grenzschutz der Schweizer Landwirtschaft angewendet werden sowie auf den einfuhrseitigen Preisausgleichmechanismus. Hinsichtlich des ausfuhrseitigen Preisausgleichmechanismus (Exportsubventionen) gilt es zu bedenken, dass sein Ziel nicht die Schaffung eines Wettbewerbsvorteils ist, sondern die Behebung eines Wettbewerbsnachteils, verursacht durch die hohen Agrarrohstoffpreise als Folge des Grenzschutzes. 
Die Kombination von Grenzschutz und Preisausgleichmechanismus hat folgende Konsequenzen: Für die Schweizer Nahrungsmittelindustrie bedeutet diese Situation eine Segmentierung des Marktes. Was ihre Produkte für den Schweizer Markt betrifft, so kann sie die durch den Grenzschutz verursachten Mehrkosten dank dem einfuhrseitigen Preisausgleich an die Konsumenten weitergeben. Gleichzeitig kann sie ihre Exportprodukte zu einem Preis im Ausland anbieten, welcher einer Situation des Freihandels entspricht, da aus ihrer Perspektive der Grenzschutz durch die Exportsubventionen neutralisiert wird. Für die Schweizer Konsumenten bedeutet diese Situation, dass sie die durch den Grenzschutz verursachten Mehrkosten tragen müssen. Für den Staat bedeutet diese Situation, dass er durch den Grenzschutz und den einfuhrseitigen Preisausgleich Zolleinnahmen einnimmt, gleichzeitig aber für die Exportsubventionen Gelder bereitstellen muss. Die Mittel, welche in die Exportsubventionen fliessen, werden schliesslich vom Schweizer Steuerzahler bezahlt. Die Landwirtschaft wiederum kann ihre Agrarrohstoffe im Inland dank dem Grenzschutz und dem Preisausgleich zu einem höheren Preis anbieten, als wenn Freihandel herrschen würde. Insgesamt übertreffen die kombinierten Kosten für die Konsumenten und die Steuerzahler die Gewinne für die Landwirtschaft. Diese Situation ist somit im Vergleich zum Freihandel gesamtvolkswirtschaftlich betrachtet wohlfahrtsmindernd.

\section{Bedeutung des Protokolls Nr. 2 für die Schweiz}

In diesem Kapitel wird die Bedeutung des Protokolls Nr. 2 für die Schweiz analysiert. Hierzu werden zunächst die Entwicklung und die Bedeutung des Handels zwischen der Schweiz und der EU im Rahmen des Protokolls Nr. 2 beleuchtet. Danach wird auf die beiden hauptsächlich betroffenen Sektoren in der Schweiz, die Landwirtschaft und die Nahrungsmittelindustrie, näher eingegangen. Schliess- 
lich wird die Bedeutung des Protokolls Nr. 2 für die Schweiz und ihre Volkswirtschaft als Ganzes diskutiert.

\subsection{Bedeutung des Handels im Rahmen des Protokolls Nr. 2}

Die Schweiz ist eine kleine offene Volkswirtschaft mit einem der höchsten Anteile des Aussenhandels am Bruttoinlandprodukt. ${ }^{48}$ Die EU ist der mit Abstand wichtigste Handelspartner der Schweiz. ${ }^{49} \mathrm{Im}$ Jahr 2010 exportierte die Schweiz Güter im Wert von rund 119,3 Mrd. Franken in die EU und importierte Güter im Wert von rund 142,1 Mrd. Franken aus der EU..$^{50} \mathrm{Im}$ selben Jahr beliefen sich die Exporte der Landwirtschaftsprodukte aus der Schweiz in die EU auf einen Wert von 4,8 Mrd. Franken, die Importe auf einen Wert von 8,8 Mrd. Franken. ${ }^{51}$ Die Exporte der Schweizer Landwirtschaftsprodukte fallen in erster Linie in die Kategorien Genussmittel (Kaffee, Schokolade etc.) und Getränke, gefolgt von Nahrungsmittelzubereitungen, Tabak und Diverses sowie Milchprodukten. ${ }^{52}$

Nachfolgend werden die wichtigsten Entwicklungen des Handels zwischen der Schweiz und der EU im Rahmen des Protokolls Nr. 2 von $2005-2011$ analysiert. ${ }^{53}$

48 BFS: „Aussenhandel - Indikatoren“, http://www.bfs.admin.ch, zuletzt besucht am 21.7.2012.

49 Integrationsbüro EDA/EVD: „Bilaterale Abkommen CH-EU, Freihandel“, http:// www.europa.admin.ch, zuletzt besucht am 21.7.2012.

50 BFS: „Ausfuhr nach Wirtschaftsräumen und Bestimmungsländern 1990-2010“, sowie „Einfuhren nach Wirtschaftsräumen und Herkunftsländern 1990-2010“, http:// www.bfs.admin.ch, zuletzt besucht am 21.7.2012.

51 BLW: „Agrarbericht 2011“, Bundesamt für Landwirtschaft, Bern. S. 12.

52 BLW: „Agrarbericht 2011“, Bundesamt für Landwirtschaft, Bern. S. 14.

53 Die Analyse basiert auf statistischen Angaben von Peter Huber (SECO), 2012. Die Angaben wurden über die Aussenhandelstatistik (swissimpex) durch Aggregation der einzelnen Tarifnummern und Zollkapitel ermittelt. 
Die Exporte von landwirtschaftlichen Verarbeitungsprodukten aus der Schweiz in die EU haben sich in den vergangenen sieben Jahren mit einer wertmässigen Zunahme von rund $91 \%$ beinahe verdoppelt und beliefen sich im Jahr 2011 auf rund 3,7 Mrd. Franken (vgl. Abbildung 3). Das Wachstum hat sich ab 2008 allerdings verlangsamt und war im Jahr 2011 sogar leicht rückläufig, was sich u.a. durch den starken Schweizer Franken erklären lässt. ${ }^{54}$

\section{In Mrd. CHF}

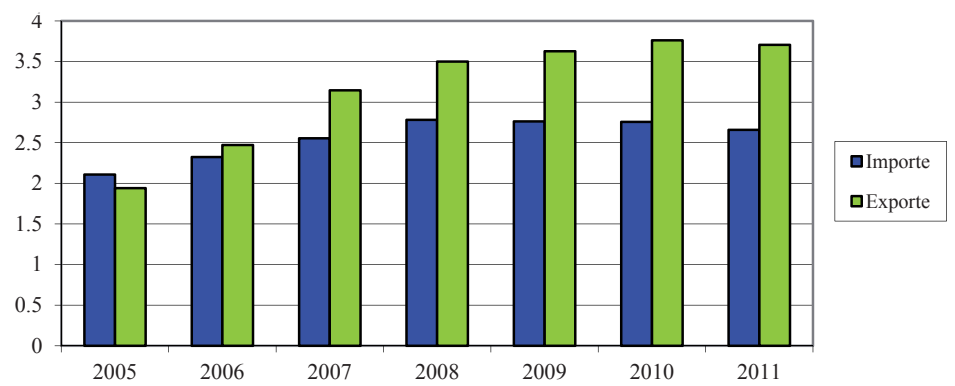

Abb. 3: Handel im Rahmen des Protokolls Nr. 2 (Tabelle I und II). Quelle: Statistische Angaben von Peter Huber (SECO), 2012. Eigene Darstellung.

Aufschlussreich ist ein Blick auf die Entwicklung der Schweizer Exporte aufgeteilt in preisausgleichberechtigte Produkte (Tabelle I) und Freihandelsprodukte (Tabelle II). Die Exporte von Freihandelsprodukten weisen eine bemerkenswerte Wachstumsrate von über 306\% auf und haben sich somit mehr als verdreifacht (vgl. Abbildung 4). Im Jahr 2011 erreichten sie einen Wert von rund 2,1 Mrd. Franken. Diese Dynamik ist in erster Linie durch den Export von Kaffee, Tee und Getränken bedingt.

54 Persönliche Auskunft von Markus Schlagenhof (SECO), 31.5.2012. 


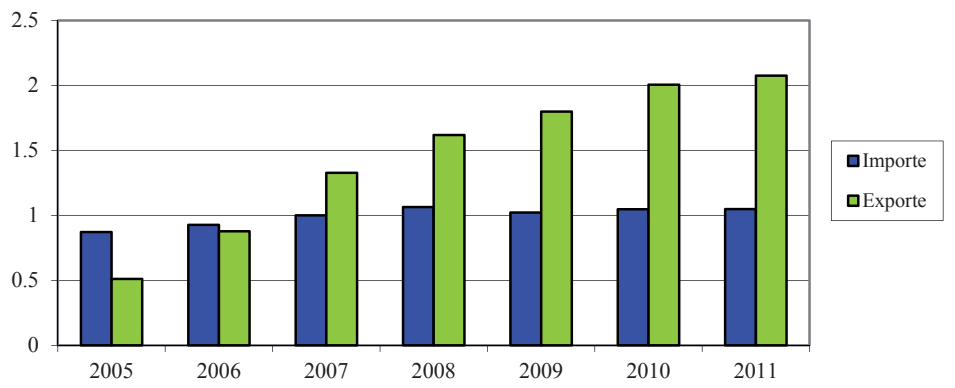

Abb. 4: Handel der Freihandelsprodukte (Tabelle II). Quelle: Statistische Angaben von Peter Huber (SECO), 2012. Eigene Darstellung.

In Bezug auf die Ausfuhren aus der Schweiz von preisausgleichberechtigen Produkten, konnte zwischen 2005 und 2011 eine Wachstumsrate von rund $14 \%$ verzeichnet werden, wobei die Ausfuhren seit 2008 leicht rückläufig sind (vgl. Abbildung 5). Im Jahr 2011 lagen die Exporte bei über 1,6 Mrd. Franken. Zu den wichtigsten Exportprodukten zählen in dieser Kategorie Schokolade, Backwaren, Kindernahrungsmittel und andere Nahrungsmittelzubereitungen.

In Mrd. CHF

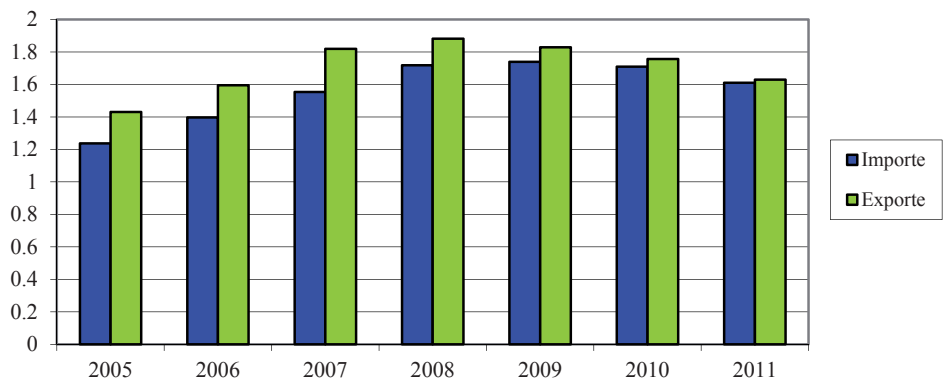

Abb. 5: Handel der preisausgleichberechtigten Produkte (Tabelle I). Quelle: Statistische Angaben von Peter Huber (SECO), 2012. Eigene Darstellung. 
Die Importe von landwirtschaftlichen Verarbeitungsprodukten aus der EU beliefen sich im Jahr 2011 auf rund 2,7 Mrd. Franken, wobei der Anteil der Freihandelsprodukte über 1 Mrd. Franken und der Anteil der preisausgleichberechtigen Produkte über 1,6 Mrd. Franken ausmachte. Die Importe aus der EU im Bereich der Freihandelsprodukte sind mit ca. 20\% im Verhältnis zu den Schweizer Exporten derselben Kategorie $(+306 \%)$ nur gering gewachsen und liegen heute auf einem deutlich tieferen Niveau. Hingegen weisen die Importe aus der EU im Bereich der preisausgleichberechtigten Produkte mit ca. 30\% ein grösseres Wachstum auf, als deren Exporte aus der Schweiz (+14\%). Im Jahr 2011 tauschten die Schweiz und die EU im ähnlichen Wertumfang von rund je 1,6 Mrd. Franken preisausgleichberechtigte Produkte.

Zusammengefasst kann folgendes festgehalten werden: Die Handelsbilanz der Schweiz mit der EU im Bereich der landwirtschaftlichen Verarbeitungsprodukte ist heute wertmässig klar positiv. Diese Situation lässt sich hauptsächlich auf die bedeutsamen Schweizer Exporte von Freihandelsgütern (Kaffee, Tee, Getränke) zurückführen. Hingegen bewegen sich die Exporte der preisausgleichberechtigten Produkten aus der Schweiz und aus der EU wertmässig im gleichen Grössenbereich. Ferner machen die Schweizer Exporte, welche in den Geltungsbereich des Protokolls Nr. 2 fallen, den Grossteil der Gesamtexporte der Landwirtschaftsprodukte in die EU aus.

\subsection{Bedeutung für die Landwirtschaft}

Um die Bedeutung des Protokolls Nr. 2 für die Schweizer Landwirtschaft zu erfassen, muss zwischen den preisausgleichberechtigten Produkten und den Freihandelsprodukten unterschieden werden. Die gegenseitige Zollfreiheit für Freihandelsprodukte tangiert die Landwirtschaft nicht direkt, da diese Produkte keine Agrarrohstoffe bein- 
halten, welche agrarpolitisch von Relevanz sind, wie insbesondere Milch und Getreide..$^{55} \mathrm{Im}$ Folgenden soll daher einzig die Bedeutung der preisausgleichberechtigten Produkte für die Landwirtschaft analysiert werden.

Reformen in der Schweizer Agrarpolitik seit Anfang der 1990-er Jahre haben dazu geführt, dass die agrarpolitische Stützung schrittweise reduziert und von der Produktion entkoppelt wurde.$^{56}$ Die Gesamtstützung der Schweizer Landwirtschaft ist heute im internationalen Vergleich jedoch nach wie vor hoch. Einerseits spielen öffentliche Gelder für Landwirtschaft und Ernährung in einem Umfang von ca.3,7 Mrd. Franken jährlich eine Rolle. ${ }^{57}$ Gemäss OECD machte die staatliche Unterstützung in der Schweiz im Jahr 2011 56\% des Einkommens der Landwirtschaft aus. ${ }^{58}$ Anderseits kommt dem Grenzschutz eine wichtige Bedeutung zu. Grenzschutzmassnahmen führen dazu, dass die Schweizer Preise für Agrarrohstoffe weitgehend vom Weltmarkt abgekoppelt sind und erheblich über dem Weltmarktpreisniveau liegen. ${ }^{59}$ Wie in Kapitel 3 aufgezeigt, trägt der Preisausgleichmechanismus dazu bei, dass die Schweizer Nahrungsmittelindustrie trotz dieser Ausgangslage fähig ist, nationale Agrarrohstoffe zu verarbeiten und ihre Produkte im Inland und im Ausland zu wettbewerbsfähigen Preisen abzusetzen.

55 Roth, Thomas (2004): „Abkommen über verarbeitete Landwirtschaftsprodukte eine neue Etappe in den Freihandelsbeziehungen mit der EU“. Die Volkswirtschaft 9.2004 .

56 Lehmann, Bernard und Lanz, Simon (2012): „Grundzüge der Agrarpolitik 20142017“. Die Volkswirtschaft 4.2012.

57 BLW:„Agrarbericht 2011“, Bundesamt für Landwirtschaft, Bern. S. 16.

58 OECD (2011): „Switzerland - Agricultural Policy Monitoring and Evaluation 2011“, http://www.oecd.org, zuletzt besucht am 21.7.2012.

59 Harder, Werner und Jung, Vinzenz (2008): „Knappe Agrarrohstoffe: Kurzfristiges Phänomen oder langfristige Herausforderung?“. Die Volkswirtschaft 9.2008. 
Die Schweizer Nahrungsmittelindustrie stellt für die Schweizer Landwirtschaft insgesamt ein wichtiger Absatzmarkt für Agrarrohstoffe dar. ${ }^{60} \mathrm{Im}$ Rahmen des Schoggigesetzes werden Ausfuhrbeiträge für insbesondere folgende Agrarrohstoffe und Zwischenprodukte gewährt, wenn diese in Form von verarbeiteten Nahrungsmitteln exportiert werden: Milch und Milchpulver, Rahm und Rahmpulver, Butter, Mehl und andere Mahlprodukte von Weizen, Dinkel, Roggen und Mengkorn, sowie Keime von Weizen, Roggen und Mengkorn. ${ }^{61}$

Am wichtigsten sind die Ausfuhrbeiträge für verarbeitete Milch- und Getreideprodukte. ${ }^{62}$ Im Jahr 2011 wurden 5,7\% der in der Schweiz produzierten Milchmenge und 9,6\% der in der Schweiz produzierten Mehlmenge im Rahmen des Schoggigesetzes als landwirtschaftliche Verarbeitungsprodukte exportiert. Der Grossteil wurde im Rahmen des Protokolls Nr. 2 in die EU exportiert und zwar 3,2\% der Milchmenge respektive $9,3 \%$ der Mehlmenge. ${ }^{63}$

Welche Bedeutung kann diesen Marktanteilen nun zugewiesen werden? Für einzelne Landwirtschaftsunternehmen im Milch- oder im Getreidesektor dürften diese Zahlen einen bedeutenden Marktanteil darstellen. Seit die Budgetmittel nicht mehr vollständig ausreichen, organisieren sich die Branchen um den aktiven Veredelungsverkehr zu verhindern (vgl. 3.2). Ziel ist es, diese Marktanteile zu halten, auch wenn damit ein geringeres Einkommen in Kauf genommen werden muss. Einerseits dürfte die Befürchtung endgültiger Marktverluste eine Rolle spielen. Gewisse Agrarrohstoffe oder Zwischenprodukte,

60 Vgl. Fial (2011): „Die Schweizer Nahrungsmittel-Industrie im 2010“, 8.2011, http:// www.fial.ch, zuletzt besucht am 21.7.2012.

61 Verordnung über die Ausfuhrbeiträge für Erzeugnisse aus Landwirtschaftsprodukten vom 23. November 2011 (SR 632.111.723), Art.1.

62 Persönliche Auskunft von Markus Schlagenhof (SECO), 31.5.2012.

63 Persönliche Auskunft von Jacques Chavaz und Kilian Greter (beide BLW), 10.7.2012. 
u.a. Butter, unterstehen dem so genannt besonderen Verfahren im Rahmen des aktiven Veredelungsverkehrs. ${ }^{64}$ Dieses Verfahren entspricht faktisch einer automatischen und generellen Bewilligung. Auch wenn die Umstellung auf den aktiven Veredelungsverkehr für die Nahrungsmittelindustrie mit einem gewissen Aufwand verbunden ist (Logistik, möglicher Einfluss auf die Rezeptur, etc.) ${ }^{65}$, dürften dafür die regulatorischen Hürden im Rahmen des besonderen Verfahrens relativ gering sein. Anderseits kann durch die aktuelle Überschussproblematik insbesondere bei Schweizer Milch und Butter die Bereitschaft erklärt werden, weswegen ein geringeres Einkommen in Kauf genommen wird. Für die Landwirtschaft dürfte es immer noch attraktiver sein, ihre Produkte zu einem reduzierten Preis auf dem Inlandmarkt zu verkaufen, anstatt zum Weltmarktpreis zu exportieren. ${ }^{66}$

Abgesehen vom oben aufgezeigten exportseitigen Effekt ist auch der importseitige Effekt des Preisausgleichs für die Landwirtschaft von Bedeutung. ${ }^{67}$ Anhand Einfuhrabgaben auf landwirtschaftliche Verarbeitungsprodukte wird sichergestellt, dass ausländische Produkte nicht aufgrund billigerer Agrarrohstoffe günstiger als inländische Produkte auf dem Schweizer Markt angeboten werden. Der Absatz jener Schweizer Agrarrohstoffe, welche schliesslich in Form von preisausgleichberechtigten Produkten auf den inländischen Markt gelangen, wird somit ebenfalls unterstützt. ${ }^{68}$

Aus der Perspektive der Landwirtschaft sind die Preisausgleichmassnahmen im Rahmen des Protokolls Nr. 2 klar von Bedeutung. Voll-

64 Zollverordnung vom 1. November 2006 (SR 631.01), Art. 170.

65 Persönliche Auskunft von Markus Schlagenhof (SECO), 31.5.2012, sowie Mathias Bergundthal (Nestlé Schweiz), 5.7.2012.

66 Persönliche Auskunft von Jacques Chavaz und Kilian Greter (beide BLW), 10.7.2012.

67 Ibid.

68 Angaben zum Umfang dieser Agrarrohstoffe liegen im Rahmen dieser Arbeit nicht vor. 
umfänglich wird dies jedoch erst ersichtlich, wenn der Preisausgleichmechanismus als Massnahme erkannt wird, um den allgemein hohen Grenzschutz der Landwirtschaft mit zu ermöglichen. Denn sollte der Preisausgleich nicht mehr durchsetzbar sein, dürfte aufgrund der Konsequenzen für die verarbeitende Nahrungsmittelindustrie der politische Druck Richtung Liberalisierung der Landwirtschaft steigen.

\subsection{Bedeutung für die Nahrungsmittelindustrie}

Wie in 5.1 aufgezeigt, exportierte die Schweizer Nahrungsmittelindustrie im Rahmen des Protokolls Nr. 2 im Jahr 2011 landwirtschaftliche Verarbeitungsprodukte im Wert von rund 3,7 Mrd. Franken. Diese Exporte sind für die betroffenen Unternehmen der Nahrungsmittelindustrie von Bedeutung, damit sie gewisse Skalenerträge realisieren können. ${ }^{69}$ Allgemein fördern geeignete Rahmenbedingungen für den Export den Produktionsstandort Schweiz. So spielte der im Abkommen über landwirtschaftliche Verarbeitungsprodukte verhandelte Freihandel für Kaffee einen mitentscheidenden Faktor für Nestlé, seinen Produktionsstandort Schweiz weiter auszubauen. ${ }^{70}$

Auch um die Bedeutung des Protokolls Nr. 2 für die Nahrungsmittelindustrie zu analysieren, gilt es zu differenzieren zwischen den preisausgleichberechtigten Produkten und den Freihandelsprodukten.

Insbesondere die Exporte von Freihandelsprodukten verzeichneten in den letzten sieben Jahren ein bedeutendes Wachstum und erreichten 2011 einen Wert von 2,1 Mrd. Franken. Diese Entwicklung lässt den Schluss zu, dass die betroffenen Produkte von grosser Bedeutung für die Schweizer Nahrungsmittelindustrie sind und auch Zukunftspotenzial aufweisen. Es gilt dabei zu bedenken, dass dieses Wachstum in

69 Persönliche Auskunft von Markus Schlagenhof (SECO), 31.5.2012.

70 Persönliche Auskunft von Mathias Bergundthal (Nestlé Schweiz), 5.7.2012. 
erster Linie auf den Export von Kaffee, Tee und Getränken zurückzuführen ist.

Die Exporte der preisausgleichberechtigen Produkte haben sich im Vergleich eindeutig weniger dynamisch entwickelt. Nichtsdestotrotz beliefen sie sich im Jahr 2011 auf einen Wert von 1,6 Mrd. Franken. Was ist nun in diesem Zusammenhang die Bedeutung des Preisausgleichmechanismus für die betroffene Schweizer Nahrungsmittelindustrie?

Im Vergleich zu einem Exportvolumen von 1,6 Mrd. Franken scheinen Ausfuhrbeiträge in der Grössenordnung von 70 Mio. Franken, wovon 2011 wiederum nur 65\% für Ausfuhren in die EU eingesetzt wurden, bescheiden. Es gilt jedoch zu beachten, dass die Margen im Detailhandel teilweise sehr gering sind, wodurch diese Ausfuhrbeiträge einen wichtigen Einfluss auf die Wettbewerbsfähigkeit der Schweizer Exporteure auf dem EU-Markt haben können. ${ }^{71}$ Zugang zu konkurrenzfähigen Rohstoffen ist ein Faktor von vielen in Bezug auf die Produktionskosten und die Wahl des Produktionsstandortes. Die Rohstoffkosten dürften mit ca. 50\% der Gesamtkosten ab Fabrik aber unbestritten eine Rolle spielen. ${ }^{72}$ Wie in Kapitel 4 aufgezeigt, werden aus der Perspektive der Nahrungsmittelindustrie die Kosten des hohen Grenzschutzes für ihre Exportprodukte durch die Exportsubventionen aufgefangen.

Auch bei der Beurteilung des Preisausgleichs für die Nahrungsmittelindustrie gilt es neben dem exportseitigen Effekt auch den importseitigen Effekt zu berücksichtigen. Importzölle auf landwirtschaftliche Verarbeitungsprodukte tragen dazu bei, dass die Schweizer Nahrungsmittelindustrie auch auf dem Schweizer Markt wettbewerbsfä-

71 Persönliche Auskunft von Markus Schlagenhof (SECO), 31.5.2012.

72 Angaben zu den Kosten: Persönliche Auskunft von Mathias Bergundthal (Nestlé Schweiz), 5.7.2012. 
hig ist. Die durch den Grenzschutz verursachten höheren Beschaffungskosten für Agrarrohstoffe können somit an die Konsumenten weitergegeben werden (vgl. Kapitel 3).

Grundsätzlich unterstützt die Schweizer Nahrungsmittelindustrie eine produzierende Landwirtschaft und bevorzugt aus Gründen der langjährigen Beziehung zu den Lieferanten, der Nachhaltigkeit und der Transportwege inländische Rohstoffe..$^{73}$ Auch die "Swissness" ist ein wichtiger Faktor, selbst wenn diese in der Konsumentenwahrnehmung im Ausland in erster Linie für die Herstellung in der Schweiz steht. ${ }^{74}$ Generell kann davon ausgegangen werden, dass die Nahrungsmittelindustrie die Verwendung von Schweizer Agrarrohstoffen bevorzugt, solange sich dies für sie wirtschaftlich gesehen rechtfertigen lässt. ${ }^{75}$ Dies dürfte unter den aktuellen Rahmenbedingungen für ihre Exportprodukte solange der Fall sein, wie der dafür zu bezahlende Preis zusammen mit den Vorteilen der Verwendung von Schweizer Agrarrohstoffen vorteilhafter als die Beschaffung von Agrarrohstoffen im aktiven Veredelungsverkehr ist. In diesem Zusammenhang gilt es zu bedenken, dass die Umstellung auf den aktiven Veredelungsverkehr für die Nahrungsmittelindustrie mit einem gewissen Aufwand verbunden ist (Logistik, möglicher Einfluss auf die Rezeptur, etc.). ${ }^{76}$ Zudem müssen gleichzeitig weiterhin nationale Agrarrohstoffe für die Produkte für den inländischen Markt bezogen werden.

Die aktuelle Situation ist allerdings aufgrund der knappen Budgetmittel mit einer gewissen Planungs- und Rechtsunsicherheit für die

73 Persönliche Auskunft von Mathias Bergundthal (Nestlé Schweiz), 5.7.2012.

74 Persönliche Auskunft von Franz Urs Schmid (Fial), 11.7.2012.

75 Persönliche Auskunft von Jacques Chavaz und Kilian Greter (beide BLW), 10.7.2012.

76 Persönliche Auskunft von Markus Schlagenhof (SECO), 31.5.2012, sowie von Mathias Bergundthal (Nestlé Schweiz), 5.7.2012. 
verarbeitende Nahrungsmittelindustrie verbunden. ${ }^{77}$ Für die Nahrungsmittelindustrie ist der Agrarfreihandel nicht zuletzt deswegen durchaus eine denkbare Alternative zur aktuellen Situation. Dadurch würden sich die Preise der Agrarrohstoffe in der Schweiz jenen im Ausland bzw. jenen in der EU angleichen und der Preisausgleichmechanismus würde überflüssig. Eine solche Entwicklung wird als Chance betrachtet. ${ }^{78}$ Somit würden klare Rahmenbedingungen herrschen und das komplizierte System von Preisausgleich, privatrechtlichen Massnahmen, Veredelungsverkehr und Importkontingenten würde hinfällig. Wichtig ist aus Perspektive der Nahrungsmittelindustrie insbesondere die Reihenfolge allfälliger Reformen. Der Preisausgleichmechanismus sollte erst dann wegfallen, wenn die Reformen in der Landwirtschaft stattgefunden haben und der Marktzugang im Ausland sichergestellt ist. ${ }^{79}$

\subsection{Gesamtvolkswirtschaftliche Bedeutung für die Schweiz}

In Bezug auf die Freihandelsprodukte kann deren gegenseitige Zollfreiheit aus volkswirtschaftlicher Perspektive als grundsätzlich positiv gewertet werden (vgl. Kapitel 4). Die folgende Analyse beschränkt sich deswegen auf den Teilaspekt der preisausgleichberechtigten Produkte.

Der Preisausgleich im Rahmen des Protokolls Nr. 2 kann als eine Konsequenz der Schweizer Agrarpolitik betrachtet werden. Um dessen Bedeutung für die Schweiz als Ganzes zu analysieren, müssen deswegen auch die Wirkungen der Agrarpolitik bedacht werden. Wie in Kapitel 4 aufgezeigt, ist der dem Protokoll Nr. 2 zu Grunde liegen-

77 Persönliche Auskunft von Mathias Bergundthal (Nestlé Schweiz), 5.7.2012, sowie von Franz Urs Schmid (Fial), 11.7.2012.

78 Ibid.

79 Persönliche Auskunft von Mathias Bergundthal (Nestlé Schweiz), 5.7.2012. 
de landwirtschaftliche Grenzschutz zusammen mit den Wirkungen des Preisausgleichmechanismus aus volkswirtschaftlicher Perspektive wohlfahrtsmindernd. Neben dem allgemeinen Wohlfahrtsverlust findet eine Umverteilung der Wohlfahrt von den Konsumenten und Steuerzahlern auf die Landwirtschaft statt.

Ferner gilt es auch die Opportunitätskosten einer solchen Politik zu bedenken. Die Schweizer Exporte sind für die Schweizer Volkswirtschaft zentral (vgl. 5.1). Somit kommt auch den Freihandelsabkommen mit anderen Partnern als der EU eine wichtige Bedeutung zu, insbesondere vor dem Hintergrund der stockenden multilateralen Verhandlungen im Rahmen der WTO. Es ist wahrscheinlich, dass die Schweiz noch mehr von Freihandelsabkommen profitieren könnte, wenn sie zu grösseren Konzessionen im Bereich der Landwirtschaft in der Lage wäre. ${ }^{80}$ Beispielsweise stellt in den aktuell laufenden Verhandlungen über ein Freihandelsabkommen mit China eine allfällige Marktöffnung in der Landwirtschaft einen höchst kontroversen Punkt dar. ${ }^{81}$ Auch was die Schweizer Exportsubventionen für landwirtschaftliche Verarbeitungsprodukte selbst betrifft, steht diesen nicht nur die EU kritisch gegenüber, sondern auch zunehmend ihre anderen Verhandlungspartner für ein Freihandelsabkommen. ${ }^{82}$

Im Vergleich zu einem umfassenden Freihandel im Agrar- und Le-

80 Abt, Marianne (2009): „Bedeutung der Freihandelsabkommen mit Partnern ausserhalb der EU“, http//:www.seco.admin.ch, zuletzt besucht am 21.7.2012.

81 Siehe z.B. Neue Zürcher Zeitung NZZ (2012): „Agrarwirtschaft als harte Nuss“, 9.7.2012, sowie Aeppli, Matteo (2012): „Freihandelsabkommen mit China - Vorteile auch für die Agrarwirtschaft“, 13.7.2012, http//:www.agrarpolitik-blog.com, zuletzt besucht am 21.7.2012.

82 Persönliche Auskunft von Peter Huber (SECO), 6.6.2012, sowie vgl. Oberzolldirektion, Sektion ZAV: „Verbot Ausfuhrbeiträge im Bereich der landwirtschaftlichen Verarbeitungsprodukte in Freihandelsabkommen mit Dritt-Ländern“, 5.6.2012, http:// www.evz.admin.ch, zuletzt besucht am 21.7.2012. 
bensmittelbereich mit der EU, bedeutet der Preisausgleichmechanismus im Rahmen des Protokolls Nr. 2 zusammen mit dem ihm zu Grunde liegenden Grenzschutz in der Landwirtschaft eine Verkleinerung der Wohlfahrt für die Schweiz. Ferner kann angenommen werden, dass generell der Grenzschutz in der Landwirtschaft zu Opportunitätskosten in der Form von suboptimalem Marktzugang für Schweizer Güter und Dienstleistungen im Rahmen von Freihandelsabkommen führt.

\subsection{Fazit}

Einerseits liegt die Vermutung nahe, dass das Protokoll Nr. 2 und dessen Preisausgleichmassnahmen basierend auf dem Schoggigesetz in erster Linie im Interesse der Landwirtschaft liegen. Auf der anderen Seite gilt es zu bedenken, dass die Landwirtschaft in der Schweiz gemäss Art. 104 der Bundesverfassung eine multifunktionale Aufgabe zu erfüllen hat. ${ }^{83}$ Hierzu gehört der Beitrag zu einer sicheren Versorgung, einer intakten Umwelt und einer dezentralen Besiedlung. Zudem soll der Bund naturnahe, umwelt- und tierfreundliche Produktionsformen fördern.

Diese multiplen Aufgaben machen offensichtlich, dass eine simple Reduktion der Wohlfahrt auf das Bruttoinlandprodukt nicht ausreicht. Letztlich geht es darum, wie viel eine Gesellschaft für eine multifunktionale Landwirtschaft zu bezahlen bereit ist und darum, die hierfür effizientesten wirtschafts- und handelspolitischen Instrumente zu identifizieren. Zudem müssen diese Instrumente national und international durchsetzbar sein. In Bezug auf das Protokoll Nr. 2 und dessen Preisausgleichmassnahmen stellt sich das drängendste Problem bei der Durchsetzbarkeit auf internationaler Ebene.

83 Bundesverfassung der Schweizerischen Eidgenossenschaft vom 18. April 1999 (SR 101), Art. 104. 


\section{Evaluation Handlungsdruck und Skizzierung möglicher Szenarien}

Die Exportsubventionen stellen das Grundproblem dar, weshalb das Protokoll Nr. 2 unter Druck steht (vgl. Kapitel 3). Heute besteht ein breiter Konsens, dass Exportsubventionen international ein Auslaufmodell darstellen und in der Zukunft abgeschafft werden müssen. Die kritische Frage betrifft demnach mögliche Alternativen zur aktuellen Situation. Diese werden im Folgenden grob umrissen dargestellt:

Aus volkswirtschaftlicher Perspektive betrachtet stellt eine Liberalisierung der Landwirtschaft und die daraus zu erwartende Anpassung der Schweizer Preise für Agrarrohstoffe an die EU bzw. Weltmarktpreise die optimale Lösung dar. Somit würden Preisausgleichmassnahmen überflüssig. In Anbetracht der Tatsache, dass die Preisausgleichmassnahmen eine Konsequenz des Agrarschutzes sind, scheint dieses Szenario logisch. Anderseits scheint es unrealistisch, dass der Abbau von Exportsubventionen für landwirtschaftliche Verarbeitungsprodukte einen solchen Grundsatzentscheid in der Agrarpolitik bewirken könnte. Dies dürfte erst im Zusammenhang mit internationalen Verpflichtungen realistisch werden. Aktuell wird eine Liberalisierung der Landwirtschaft in erster Linie in einem Freihandelsabkommen mit der EU (FHAL), oder im Rahmen der WTO-Doha-Runde angestrebt. Sowohl das FHAL als auch die Doha-Runde sind jedoch ins Stocken geraten und mit raschen Fortschritten ist in beiden Bereichen nicht zu rechnen.

Es ist also wahrscheinlich, dass der Abbau der Exportsubventionen den in absehbarer Zeit grösseren Handlungsdruck darstellt als die Liberalisierung der Landwirtschaft. Konkret würde dies bedeuten, dass zumindest der ausfuhrseitige Preisausgleich im Rahmen des Schoggigesetzes und somit auch im Rahmen des Protokolls Nr. 2 in Zukunft nicht mehr haltbar wäre. Wenn nun gleichzeitig keine Libe- 
ralisierung der Landwirtschaft möglich ist, was wären dann mögliche Szenarien?

Ein erstes Szenario betrifft die Ausweitung des aktiven Veredelungsverkehrs im Sinne einer Teilliberalisierung. Dies würde in erster Linie den Verlust von Marktanteilen für die Landwirtschaft bedeuten. Ein weiteres Szenario wäre die Ausweitung der privatrechtlichen Massnahmen. Dass die Übernahme der gesamten Preisdifferenz durch die Branche aber machbar wäre, ist unwahrscheinlich. Eher realistisch wäre daher eine Kombination der Szenarien 1 und 2, d.h., eine gleichzeitige Ausweitung des aktiven Veredelungsverkehrs und der privatrechtlichen Massnahmen. Ein letztes Szenario, bzw. eine weitere mögliche Konsequenz, stellt die Abwanderung von Vertretern der Nahrungsmittelindustrie ins Ausland dar. ${ }^{84}$

\section{Schlussfolgerungen}

Die Handelsbilanz der Schweiz mit der EU im Bereich der landwirtschaftlichen Verarbeitungsprodukte, welche in den Geltungsbereich des Protokolls Nr. 2 fallen, ist heute wertmässig klar positiv. Diese Situation ist in erster Linie das Resultat der bedeutsamen Schweizer Exporte im Bereich der Freihandelsprodukte. Die Schweizer Exporte der preisausgleichberechtigten Produkte haben sich im Vergleich weniger dynamisch entwickelt.

Bei der Beurteilung der Bedeutung des Protokolls Nr. 2 für die Schweiz, muss zwischen den Freihandelsprodukten und den preisausgleichberechtigten Produkten differenziert werden. Was die erste Kategorie betrifft, so kann deren gegenseitige Zollfreiheit aus volkwirtschaftlicher

84 Siehe z.B. Neue Zürcher Zeitung NZZ (2009): „Nestlé warnt vor Fabrikschliessungen“", 19.4.2009. 
Perspektive als grundsätzlich positiv gewertet werden. Der Handel mit den preisausgleichberechtigten Produkten bringt den Einsatz von Einfuhrabgaben (Importzöllen) und Ausfuhrbeiträgen (Exportsubventionen) durch die Schweiz mit sich. Diese handelspolitischen Massnahmen können als eine Konsequenz der Schweizer Agrarpolitik und den daraus resultierenden hohen Agrarrohstoffpreisen gewertet werden. Im Vergleich zu einem umfassenden Freihandel im Agrar- und Lebensmittelbereich mit der EU bedeutet der Preisausgleichmechanismus im Rahmen des Protokolls Nr. 2 zusammen mit dem ihm zu Grunde liegenden Grenzschutz in der Landwirtschaft eine Verkleinerung der Wohlfahrt für die Schweiz. Neben dem allgemeinen Wohlfahrtsverlust findet eine Umverteilung der Wohlfahrt von den Konsumenten und Steuerzahlern auf die Landwirtschaft statt.

Die Aufrechterhaltung des Preisausgleichs im Rahmen des Protokolls Nr. 2 ist in erster Linie für die Landwirtschaft von Bedeutung. Die Nahrungsmittelindustrie bevorzugt die Verwendung von Schweizer Agrarrohstoffen, solange sich dies für sie wirtschaftlich gesehen rechtfertigen lässt. Insbesondere wenn Schweizer Agrarrohstoffe in Zukunft nicht mehr ausreichend und zu einem wettbewerbsfähigen Preis erhältlich sein sollten, stellt aus Perspektive der Nahrungsmittelindustrie der Freihandel im Agrar- und Lebensmittelbereich eine chancenreiche Alternative zur aktuellen Situation dar.

Die Zukunft des Protokolls Nr. 2 ist hauptsächlich aufgrund der darin vorgesehenen Exportsubventionen im Rahmen des Preisausgleichmechanismus fraglich. Es besteht ein breiter Konsens, dass Exportsubventionen international ein Auslaufmodell darstellen, insbesondere aufgrund der WTO-Ministererklärung von Hongkong von 2005. Die Budgetknappheit im Rahmen des Schoggigesetzes sowie die kritische Haltung der EU gegenüber den schweizerischen Exportsubventionen für landwirtschaftliche Verarbeitungsprodukte stellen eine weitere Herausforderung für die Zukunft des Protokolls Nr. 2 dar. 
Die Frage möglicher Alternativen zur aktuellen Situation ist heikel und weitreichend, da eine Diskussion über eine künftige Abschaffung dieser Exportsubventionen an die Diskussion über eine künftige Liberalisierung der Schweizer Landwirtschaft gekoppelt ist. Mögliche Alternativen reichen von einer grundsätzlichen Liberalisierung der Landwirtschaft, über eine Teilliberalisierung im Rahmen einer Ausweitung des aktiven Veredelungsverkehrs, über eine Ausdehnung der privatrechtlichen Massnahmen, bis hin zu einer Abwanderung von Vertretern der Nahrungsmittelindustrie ins Ausland.

Aus volkswirtschaftlicher Perspektive betrachtet, stellt eine Liberalisierung der Landwirtschaft und die daraus zu erwartende Anpassung der Schweizer Preise für Agrarrohstoffe an die EU bzw. an die Weltmarktpreise die optimale Lösung dar. Somit würden Preisausgleichmassnahmen überflüssig. Eine solch weitgehende Reform in der Schweizer Agrarpolitik dürfte aber erst im Rahmen internationaler Verpflichtungen realistisch werden. Solch ein Szenario wiederum scheint heute in weiter Ferne, da in absehbarere Zeit mit keinen grossen Fortschritten in den relevanten Verhandlungen im Rahmen der WTO, sowie im Rahmen eines Freihandelsabkommen im Agrar- und Lebensmittelbereich mit der EU, zu rechnen ist. Der Handlungsdruck für eine gänzliche Abschaffung aller Exportsubventionen dürfte hingegen anhalten und womöglich steigen, womit eine Ausweitung des aktiven Veredelungsverkehrs allenfalls verbunden mit einer Ausweitung der privatrechtlichen Massnahmen durch die Branche ein realistisches Szenario darstellt. Die Nahrungsmittelindustrie würde für ihre Exportprodukte ausländische Agrarrohstoffe zollfrei importieren und für ihre Produkte für den inländischen Markt weiterhin Schweizer Agrarrohstoffe verwenden. Dieses Szenario würde in erster Linie einen gewissen Marktanteilverlust und allenfalls eine gewisse Preissenkung für die Schweizer Landwirtschaft bedeuten, sowie im internationalen Vergleich anhaltend hohe Kosten für die Schweizer Konsumenten von preisausgleichberechtigten Produkten. 


\section{Literaturverzeichnis}

Abt, Marianne (2009): „Bedeutung der Freihandelsabkommen mit Partnern ausserhalb der EU", http://www.seco.admin.ch/themen/00513/00515/01330/index.html?lang=de, zuletzt besucht am 21.7.2012.

Aeppli, Matteo (2012): „Freihandelsabkommen mit China - Vorteile auch für die Agrarwirtschaft", 13.7.2012, http://agrarpolitik-blog. com/2012/07/13/freihandelsabkommen-mit-china-vorteile-auch-furdie-agrarwirtschaft/, zuletzt besucht am 21.7.2012.

BFS (Bundesamt für Statistik): „Ausfuhr nach Wirtschaftsräumen und Bestimmungsländern 1990-2010“ http://www.bfs.admin.ch/bfs/ portal/de/index/themen/06/05/blank/data.html, zuletzt besucht am 21.7.2012.

BFS (Bundesamt für Statistik):: „Aussenhandel - Indikatoren“, http:// www.bfs.admin.ch/bfs/portal/de/index/themen/06/05/blank/key/handelsbilanz.html, zuletzt besucht am 21.7.2012.

BFS (Bundesamt für Statistik):: „Einfuhren nach Wirtschaftsräumen und Herkunftsländern 1990-2010", http://www.bfs.admin.ch/bfs/ portal/de/index/themen/06/05/blank/data.html, zuletzt besucht am 21.7.2012.

BLW (Bundesamt für Landwirtschaft): „Agrarbericht 2011“, Bundesamt für Landwirtschaft, Bern.

BO Milch (Branchenorganisation Milch): „Interventionsfond“, http:// www.ip-lait.ch/site/de/interventionsfonds.html, zuletzt besucht am 21.7.2012. 
Fial (Föderation der Schweizerischen Nahrungsmittelindustrien) (2011): „Die Schweizer Nahrungsmittel-Industrie im 2010“, 8.2011, http://www.fial.ch/de/statistics/, zuletzt besucht am 21.7.2012.

Harder, Werner und Jung, Vinzenz (2008): „Knappe Agrarrohstoffe: Kurzfristiges Phänomen oder langfristige Herausforderung?". Die Volkswirtschaft 9.2008.

Integrationsbüro EDA/EVD: „Bilaterale Abkommen CH-EU, Freihandel", http://www.europa.admin.ch/themen/00500/00506/00526/index. html?lang=de, zuletzt besucht am 21.7.2012.

Integrationsbüro EDA/EVD (2011): „Landwirtschaftliche Verarbeitungsprodukte: Informationsblatt", 8.2011, http://www.europa.admin. ch/themen/00500/00506/00512/index.html?lang=de, zuletzt besucht am 21.7.2012.

Krugman, Paul / Obstfeld, Maurice (2000): „International Economics, Theory and Policy, Fifth Edition", Addison-Wesley.

Lehmann, Bernard und Lanz, Simon (2012): „Grundzüge der Agrarpolitik 2014-2017“. Die Volkswirtschaft 4.2012.

Neue Zürcher Zeitung NZZ (2012): „Agrarwirtschaft als harte Nuss“, 9.7.2012.

Neue Zürcher Zeitung NZZ (2009): „Ehrgeizige Verhandlungsziele für EU-Agrarabkommen“, 27.6.2009.

Neue Zürcher Zeitung NZZ (2009): „Nestlé warnt vor Fabrikschliessungen“, 19.4.2009. 
Oberzolldirektion, Sektion ZAV: „Verbot Ausfuhrbeiträge im Bereich der landwirtschaftlichen Verarbeitungsprodukte in Freihandelsabkommen mit Dritt-Ländern“, 5.6.2012, http://www.ezv.admin.ch/zollinfo firmen/besonderheiten/00388/index.html?lang=de, zuletzt besucht am 21.7.2012.

OECD (2011): „Switzerland - Agricultural Policy Monitoring and Evaluation 2011", http://www.oecd.org/document/34/0,3746, en 26493740148709218111 1 37401,00.html, zuletzt besucht am 21.7.2012.

Roth, Thomas (2004): „Abkommen über verarbeitete Landwirtschaftsprodukte - eine neue Etappe in den Freihandelsbeziehungen mit der EU“. Die Volkswirtschaft 9.2004.

Schweizer Bauer (2009): „Einigung bei Rohstoffpreisausgleich“, 19.12.2009.

Tinner, Willy (2006) „Landwirtschaftliche Verarbeitungserzeugnisse: Würdigung des Abkommens", in Kaddous, Christine / Jametti Greiner, Monique (Hrsg.): Bilaterale Abkommen II Schweiz-EU und andere neue Abkommen. Genf, Basel (etc.): Helbling \& Lichtenhahn (etc.), S. 657-674.

WTO: "Goods schedule annexed to Marrakesh Protocol, Liste LIX (59)", CHE4-201, http://www.wto.org/english/tratop e/schedules e/ goods schedules table e.htm, zuletzt besucht am 21.7.2012.

WTO: "Ministerial Declaration adopted on 18 December 2005", http:// www.wto.org/english/thewto_e/minist_e/min05_e/final_text_e.htm, zuletzt besucht am 21.7.2012. 
Wyss-Aerni, Roland (2008): „Rohstoffverbilligung kommt die Bauern teuer", Landwirtschaftlicher Informationsdienst LID, 31.3.2008.

\section{Gesetzestexte und Rechtssachen}

Abkommen vom 26. Oktober 2004 zwischen der Schweizerischen Eidgenossenschaft und der Europäischen Gemeinschaft zur Änderung des Abkommens zwischen der Schweizerischen Eidgenossenschaft und der Europäischen Wirtschaftsgemeinschaft vom 22. Juli 1972 in Bezug auf die Bestimmungen über landwirtschaftliche Verarbeitungserzeugnisse (SR 0.632.401.23).

Botschaft zur Genehmigung der bilateralen Abkommen zwischen der Schweiz und der Europäischen Union, einschliesslich der Erlasse zur Umsetzung der Abkommen („Bilaterale II“) vom 1. Oktober 2004. In Bundesblatt Nr. 44, 9. November 2004, S. 5965-6300.

Bundesgesetz vom 13. Dezember 1974 über die Ein- und Ausfuhr von Erzeugnissen aus Landwirtschaftsprodukten (SR 632.111.72).

Bundesverfassung der Schweizerischen Eidgenossenschaft vom 18. April 1999 (SR 101).

Interpellation 10.3735 Grin Jean-Pierre: „Zukunft des „Schoggigesetzes“, Antwort des Bundesrates vom 17.11.2010.

Motion 10.3818 Darbellay Christophe: „Verhandlungen mit der EU über ein Freihandelsabkommen im Agrar- und Lebensmittelbereich stoppen“, eingereicht am 1.10.2010. 
Motion 12.3014 Kommission für Wirtschaft und Abgaben SR: „Kontrollierte Öffnung im Agrarbereich statt Verhandlungsverbot", eingereicht am 14.2.2012.

Protokoll Nr. 2 vom 22. Juli 1972 über bestimmte landwirtschaftliche Verarbeitungserzeugnisse (SR 0.632.401.2).

Verordnung über die Ausfuhrbeiträge für Erzeugnisse aus Landwirtschaftsprodukten vom 23. November 2011 (SR 632.111.723).

Zollgesetz vom 18. März 2005 (SR 631.0).

Zollverordnung vom 1. November 2006 (SR 631.01).

\section{Interviews und persönliche Kontakte}

Bundesamt für Landwirtschaft BLW:

Jacques Chavaz, stv. Direktor

Kilian Greter, wissenschaftlicher Mitarbeiter Fachbereich internationale Handelspolitik

Föderation der Schweizerischen Nahrungsmittelindustrien Fial:

Franz Urs Schmid, Co-Geschäftsführer

Integrationsbüro IB:

David Keller, wissenschaftlicher Mitarbeiter Wirtschafts- und Finanzfragen

Nestlé Schweiz:

Mathias Bergundthal, Aussenhandelsexperte 
Staatssekretariat für Wirtschaft SECO:

- Peter Huber, wissenschaftlicher Mitarbeiter Ressort internationaler Warenverkehr

- Christine Lewis, wissenschaftliche Mitarbeiterin Ressort WTO

- Markus Schlagenhof, Ressortleiter internationaler Warenverkehr

- Remigi Winzap, Ressortleiter WTO 


\section{BASLER SCHRIFTEN ZUR EUROPÄISCHEN INTEGRATION}

Ca. 4 x jährlich bringt das Europainstitut eine „Basler Schrift für europäische Integration" heraus.

Diese steht ab der Nr. 50 unter http://europa.unibas.ch/dokumentation/ basler-schriften/ als kosteloser Download zur Verfügung.

Sie kann ausserdem als Broschüre beim Europainstitut direkt bestellt werden.

Wir bestellen die Schriftenreihe im Jahresabonnement zu CHF 120.-. Das Abonnement verlängert sich automatisch um ein Jahr, wenn es nicht drei Monate vor Ablauf schriftlich gekündigt wird.

O Wir bestellen folgende Nummern zum Preis von CHF 20.- (Doppelnr. 30.-)

O Wir sind an einem Publikationsaustausch interessiert.

O Wir sind an Weiterbildungs-Unterlagen (Nachdiplomkurs) interessiert.

* vergriffen

O Nr. 1 Subsidiarität - Schlagwort oder Kurskorrektur (mit Beiträgen von Flavio Cotti, Jean-Paul Heider, Jakob Kellenberger und Erwin Teufel) (Doppelnummer).

O Nr. 2 Ein schweizerisches Börsengesetz im europäischen Kontext (Tagungsband/Doppelnummer).

O Nr. 3 Martin Holland, The European Union's Common Foreign and Security Policy: The Joint Action Towards South Africa. ${ }^{*}$

O Nr. 4 Brigid Gavin, The Implications of the Uruguay Round for the Common Agricultural Policy.

O Nr. 6 Urs Saxer, Die Zukunft des Nationalstaates.

O Nr. 7 Frank Emmert, Lange Stange im Nebel oder neue Strategie? Die aktuelle Rechtsprechung des EuGH zur Warenverkehrsfreiheit. 
O Nr. 8 Stephan Kux, Subsidiarity and the Environment: Implementing International Agreements.

O Nr. 9 Arbeitslosigkeit (mit Beiträgen von Christopher Boyd, Wolfgang Franz und Jean-Luc Nordmann).

O Nr. 10 Peter Schmidt, Die aussenpolitische Rolle Deutschlands im neuen Europa.

O Nr. 11 Hans Baumann, Möglichkeiten und Grenzen der Sozialen Dimension nach Maastricht: Das Beispiel der Bauwirtschaft.*

O Nr. 12 Georg Kreis, Das schweizerische Staatsvertragsreferendum: Wechselspiel zwischen indirekter und direkter Demokratie.

O Nr. 13 Markus Lusser, Die europäische Währungsintegration und die Schweiz.

O Nr. 14 Claus Leggewie, Ist kulturelle Koexistenz lernbar?

O Nr. 15 Rolf Lüpke, Die Durchsetzung strengerer einzelstaatlicher Umweltschutznormen im Gemeinschaftsrecht (Doppelnummer).

O Nr.16 Stephan Kux, Ursachen und Lösungsansätze des Balkankonflikts: Folgerungen für das Abkommen von Dayton.

O Nr. 17 Jan Dietze/Dominik Schnichels, Die aktuelle Rechtssprechung des Europäischen Gerichtshofes zum Europäischen Gerichtsstands- und Vollstreckungsübereinkommen (EuGVÜ).

O Nr. 18 Basler Thesen für die künftige Verfassung Europas (2. Aufl.).

O Nr. 19 Christian Garbe, Subsidiarity and European Environmental Policy: An Economic Perspective.

O Nr. 20 Claudia Weiss, Die Schweiz und die Europäische Menschenrechtskonvention: Die Haltung des Parlaments 1969-1995.

O Nr. 21 Gunther Teubner, Globale Bukowina: Zur Emergenz eines transnationalen Rechtspluralismus.

O Nr. 22 Jürgen Mittelstrass, Stichwort Interdisziplinarität (mit einem anschliessenden Werkstattgespräch). 
O Nr. 23 William James Adams, The Political Economy of French Agriculture.

O Nr. 24 Aktuelle Fragen der Wirtschafts- und Währungsunion (mit Beiträgen von Gunter Baer, Peter Bofinger, Renate Ohr und Georg Rich) (Tagungsband/Doppelnummer). ${ }^{*}$

O Nr. 25 Franz Blankart, Handel und Menschenrechte.

O Nr. 26 Manfred Dammeyer/Christoph Koellreuter, Die Globalisierung der Wirtschaft als Herausforderung an die Regionen Europas.

O Nr. 27 Beat Sitter-Liver, Von Macht und Verantwortung in der Wissenschaft.*

O Nr. 28 Hartwig Isernhagen, Interdisziplinarität und die gesellschaftliche Rolle der Geistes- und Kulturwissenschaften.

O Nr. 29 Muriel Peneveyre, La réglementation prudentielle des banques dans l'Union Européenne.

O Nr. 30 Giuseppe Callovi/Roland Schärer/Georg Kreis, Citoyenneté et naturalisations en Europe.

O Nr. 31 Peter Häberle, Gemeineuropäisches Verfassungsrecht.

O Nr. 32 Jacques Pelkmans, Europe's Rediscovery of Asia. Political, economic and institutional aspects.

O Nr. 33 Maya Krell, Euro-mediterrane Partnerschaft. Die Chancen des Stabilitätstransfers. *

O Nr. 34 Valéry Giscard d'Estaing, L'Union Européenne: Elargissement ou approfondissement?

O Nr. 35 Martin Holland, Do Acronyms Matter? The Future of ACP-EU Relations and the Developing World. ${ }^{*}$

O Nr. 36 Andreas Guski, Westeuropa - Osteuropa: Aspekte einer problematischen Nachbarschaft.

O Nr. 37 Matthias Amgwerd, Autonomer Nachvollzug von EU-Recht durch die Schweiz - unter spezieller Berücksichtigung des Kartellrechts (Doppelnr.). 
O Nr. 38 Manfred Rist, Infotainment oder Sachinformation? Die Europäische Union als journalistische Herausforderung (Doppelnummer).

O Nr. 39 Lothar Kettenacker/Hansgerd Schulte/Christoph Weckerle, Kulturpräsenz im Ausland. Deutschland, Frankreich, Schweiz.

O Nr. 40 Georg Kreis/Andreas Auer/Christoph Koellreuter, Die Zukunft der Schweiz in Europa? Schweizerische Informationstagung vom 15. April 1999 veranstaltet durch das EUROPA FORUM LUZERN.

O Nr. 41 Charles Liebherr, Regulierung der audiovisuellen Industrie in der Europäischen Union.

ONr. 42/3 Urs Saxer, Kosovo und das Völkerrecht. Ein Konfliktmanagement im Spannungsfeld von Menschenrechten, kollektiver Sicherheit und Unilateralismus.

O Nr. 44/5 Gabriela Arnold, Sollen Parallelimporte von Arzneimitteln zugelassen werden? Eine Analyse der Situation in der Europäischen Union mit Folgerungen für die Schweiz.*

O Nr. 46 Markus Freitag, Die politischen Rahmenbedingungen des

Euro: Glaubwürdige Weichenstellungen oder Gefahr möglicher Entgleisungen?

O Nr. 47/8 Andrew Watt, "What has Become of Employment Policy?"

- Explaining the Ineffectiveness of Employment Policy in the European Union.

O Nr. 49 Christian Busse, Österreich contra Europäische Union - Eine rechtliche Beurteilung der Reaktionen der EU und ihrer Mitgliedstaaten auf die Regierungsbeteiligung der FPÖ in Österreich.

O Nr. 50 Thomas Gisselbrecht, Besteuerung von Zinserträgen in der Europäischen Union - Abschied vom Schweizerischen Bankgeheimnis? 
O Nr. 51 Uta Hühn, Die Waffen der Frauen: Der Fall Kreil - erneuter Anlass zum Konflikt zwischen europäischer und deutscher Gerichtsbarkeit? EuGH, Urteil vom 11.1.2000 in der Rs. C-283/98, Tanja Kreil/BRD.

O Nr. 52/3 Thomas Oberer, Die innenpolitische Genehmigung der bilateralen Verträge Schweiz - EU: Wende oder Ausnahme bei aussenpolitischen Vorlagen?

O Nr. 54 Georg Kreis, Gibraltar: ein Teil Europas - Imperiale oder nationale Besitzansprüche und evolutive Streiterledigung.

O Nr. 55 Beat Kappeler, Europäische Staatlichkeit und das stumme Unbehagen in der Schweiz. Mit Kommentaren von Laurent Goetschel und Rolf Weder.

O Nr. 56 Gürsel Demirok, How could the relations between Turkey and the European Union be improved?

O Nr. 57 Magdalena Bernath, Die Europäische Politische Gemeinschaft. Ein erster Versuch für eine gemeinsame europäische Aussenpolitik.

O Nr. 58 Lars Knuchel, Mittlerin und manches mehr. Die Rolle der Europäischen Kommission bei den Beitrittsverhandlungen zur Osterweiterung der Europäischen Union. Eine Zwischenbilanz.

O Nr. 59 Perspektiven auf Europa. Mit Beiträgen von Hartwig Isernhagen und Annemarie Pieper.

O Nr. 60 Die Bedeutung einer lingua franca für Europa. Mit Beiträgen von Georges Lüdi und Anne Theme.

O Nr. 61 Felix Dinger, What shall we do with the drunken sailor? EC Competition Law and Maritime Transport.

O Nr. 62 Georg F. Krayer, Spielraum für Bankegoismen in der EUBankenordnung.

O Nr. 63 Philippe Nell, China's Accession to the WTO: Challenges ahead. 
O Nr. 64 Andreas R. Ziegler, Wechselwirkung zwischen Bilateralismus und Multilateralismus. Das Beispiel der Freihandelsabkommen der EFTA-Staaten.

O Nr. 65 Markus M. Haefliger, Die europäische Konfliktintervention im burundischen Bürgerkrieg, („Arusha-Prozess“) von 1996 bis 2002.

O Nr. 66 Georg Kreis (Hrsg), Orlando Budelacci (Redaktion): Der Beitrag der Wissenschaften zur künftigen Verfassung der EU. Interdisziplinäres Verfassungssymposium anlässlich des 10 Jahre Jubiläums des Europainstituts der Universität Basel.

O Nr. 67 Francis Cheneval, Die Europäische Union und das Problem der demokratischen Repräsentation.

O Nr. 68 Politik und Religion in Europa. Mit Beiträgen von Orlando Budelacci und Gabriel N. Toggenburg. *

O Nr. 69 Chantal Delli, Das Wesen der Unionsbürgerschaft, Überlegungen anhand des Falls Rudy Grzelczyk gegen Centre public d'aide sociale d'Ottignies-Louvain-la-Neuve.

O Nr. 70 Federica Sanna, La garantie du droit de grève en Suisse et dans la I'Union européenne.

O Nr. 71 Elze Matulionyte, Transatlantic GMO Dispute in the WTO: Will Europe further abstain from Frankenstein foods?

ONr. 72 Rolf Weder (Hrsg.), Parallelimporte und der Schweizer Pharmamarkt.

O Nr. 73 Marc Biedermann, Braucht die EU Lobbying-Gesetze? Annäherungen an eine schwierige Frage.

O Nr. 74 Hanspeter K. Scheller, Switzerland's Monetary Bilateralism. The episode of 1975.

O Nr. 75 Bruno Kaufmann/Georg Kreis/Andreas Gross, Direkte Demokratie und europäische Integration. Die Handlungsspielräume der Schweiz. 
O Nr. 76 Daniel Zimmermann, Die Zwangslizenzierung von Immaterialgüterrechten nach Art. $82 \mathrm{EG}$. Schutz vor Wettbewerb oder Schutz des Wettbewerbs?

O Nr. 77 Laurent Goetschel/Danielle Lalive d'Epinay (Hrsg.), Die Beziehungen zwischen Wirtschaft und Aussenpolitik: Eine natürliche Symbiose?

O Nr. 78 Georg Kreis (Hrsg.), Frieden und Sicherheit. Israel und die Nahostkrise als europäisches Thema. *

O Nr. 79 Christoph Nufer, Bilaterale Verhandlungen, wie weiter? Liberalisierung der Dienstleistungen zwischen der Schweiz und der EU: Gewinner und Verlierer aus Schweizer Sicht.

O Nr. 80 Simon Marti, Die aussenpolitischen Eliten der Schweiz und der Beginn der europäischen Integration. Eine rollentheoretische Untersuchung der schweizerischen Teilnahme an der Lancierung des Marshallplans und an der Errichtung der Organisation für wirtschaftliche Zusammenarbeit in Europa 1947-1948.

O Nr. 81 Thomas Cottier, Rachel Liechti, Die Beziehungen der Schweiz zur Europäischen Union: Eine kurze Geschichte differenzieller und schrittweiser Integration / Thomas Cottier, Alexandra Dengg: Der Beitrag des freien Handels zum Weltfrieden.

O Nr. 82 Martina Roth, Die neue Rolle Nicht-Staatlicher Organisationen. Möglichkeiten und Grenzen am Beispiel des Engagements in der Konflikt-Prävention; Gereon Müller-Chorus: Privatwirtschaftliche Organisation der Trinkwasserversorgung. Fluch oder Segen?

O Nr. 83 Christoph A. Spenlé (Hrsg.), Die Europäische Menschenrechtskonvention und die nationale Grundrechtsordnung. Spannungen und gegenseitige Befruchtung.

O Nr. 84 Peter Maurer, Europa als Teil der UNO.

O Nr. 85 Georg Kreis (Hrsg.), Europa als Museumsobjekt. 
O Nr. 86 Christa Tobler (Hrsg.), Aspekte des Finanzdienstleistungs- und Unternehmenssteuerrechts nach dem EG-Recht und dem bilateralen Recht.

O Nr. 87 Christa Tobler, Internetapotheken im europäischen Recht. Positive und negative Integration am Beispiel des grenzüberschreitenden Verkaufs von Arzneimitteln in der EU und in der Schweiz.

O Nr. 88 Ausra Liepinyte, Emigration von Arbeitskräften nach dem EU-Beitritt: wer gewinnt und wer verliert? Eine Analyse am Fallbeispiel Lettlands.

O Nr. 89 Laurent Goetschel/Daniel Michel, Der aussenpolitische Handlungsspielraum der Schweiz als Nichtmitglied der Europäischen Union: ein Blick auf einige Aspekte der Friedensförderung.

O Nr. 90 Martin Alioth, Irland und Europa 2002- 2009.

O Nr. 91 Sarah Conradt, Die Wirtschaftskrise - eine Chance für die Umwelt?

O Nr. 92 Sara Behrend, Möglichkeiten und Auswirkungen einer Abkehr von der nationalen Erschöpfung für Pharmaprodukte aus Sicht der Schweiz und einkommensschwacher Länder.

O Nr. 93 Roland Vaubel, Rechtfertigt die Bankenkrise die Bankenregulierungen der Europäischen Union?

O Nr. 94 Georg Kreis, Etappen auf dem Weg der französisch-deutschen Verständigung bis 1963 - mit einem Ausblick auf die Zeit danach.

O Nr. 95 Michael Ambühl, Interessendifferenzen und Interessenkongruenzen in den Beziehungen zur EU - mit Fokus auf Finanzfragen; Michael Reiterer, Die Beziehungen zwischen der EU und der Schweiz "dynamisieren“ oder die "Grenzen des Bilateralismus". 
O Nr. 96 Johannes Kohlschütter, Lokomotiven der Einigung - Europäische Eisenbahnen zwischen Kooperation und Konkurrenz.

O Nr. 97 Aline Müller, The Future of EFTA from a Swiss point of View.

O Nr. 98 Elena Mirkovska, Solar energy: Impetus for the future development of the Macedonian economy.

O Nr. 99 Christine Breitschmid, Agrarfreiheit Schweiz - EU. Analyse vor dem Hintergrund der Käsemarkt-Liberalisierung.

O Nr. 100 Georg Kreis (Hg.), Grenzüberschreitende Mikrointegration Der Basler Dreiländerraum gestern-heute-morgen.

O Nr. 101 Nils Baier, Die Medienpolitik der EU. Die Medien im Spannungsfeld zwischen Liberalisierung und Rolle als Vierte Gewalt in einer Demokratie. 
ISBN-13: 978-3-905751-27-7

ISBN-10: 3-905751-27-5

Europainstitut der Universität Basel, Gellertstrasse 27, CH-4020 Basel, Schweiz, Tel. ++41 (0) 6131797 67, FAX ++41 (0) 613179766 E-mail: europa@unibas.ch, Internet: www.europa.unibas.ch

(c) Europainstitut der Universität Basel 2013

Alle Rechte vorbehalten. Nachdruck, auch auszugsweise, sowie die Verbreitung auf elektronischem, photomechanischem oder sonstigem Wege bedürfen einer schriftlichen Genehmigung des Europainstituts. 